\title{
Increasing phagocytosis of microglia through targeting CD33 with liposomes displaying glycan ligands
}

\begin{abstract}
Abhishek Bhattacherjee ${ }^{1}$, Gour Chand Daskhan ${ }^{1}$, Arjun Bains ${ }^{1}$, Adrianne E. S. Watson ${ }^{2}$, Ghazaleh Eskandari-Sedighi ${ }^{1}$, Chris D. St. Laurent ${ }^{1}$, Anastassia Voronova ${ }^{2}$, and Matthew S. Macauley ${ }^{1,3,{ }^{*}}$
\end{abstract}

${ }^{1}$ Department of Chemistry, University of Alberta. Edmonton, Alberta, Canada

${ }^{2}$ Department of Medical Genetics, University of Alberta. Edmonton, Alberta, Canada

${ }^{1}$ Department of Medical Microbiology and Immunology, University of Alberta. Edmonton, Alberta, Canada

*Corresponding author: address: 11227 Saskatchewan Dr., Edmonton, Alberta, T6G 2G2; email: macauley@ualberta.ca 


\section{Abstract}

CD33 is an immunomodulatory receptor expressed on microglia and genetically linked to Alzheimer's disease (AD) susceptibility. While antibodies targeting CD33 have entered clinical trials to treat neurodegeneration, it is unknown whether the glycan-binding properties of CD33 can be exploited to modulate microglia. Here, we use liposomes that multivalently display glycan ligands of CD33 (CD33L liposomes) to engage CD33. We find that CD33L liposomes increase phagocytosis of cultured monocytic cells and microglia in a CD33-dependent manner. Enhanced phagocytosis strongly correlates with loss of CD33 from the cell surface and internalization of liposomes. Increased phagocytosis by treatment with CD33L liposomes is dependent on a key intracellular signaling motif on CD33 as well as the glycan-binding ability of CD33. These effects are specific to trans engagement of CD33 by CD33L liposomes, as cis engagement through insertion of lipid-linked CD33L into cells produces the opposite effect on phagocytosis. Moreover, intracerebroventricular injection of CD33L liposomes in mice enhances phagocytosis of microglia in a CD33-dependent manner. These results demonstrate that multivalent engagement of CD33 with glycan ligands can modulate microglial cell function. 


\section{Introduction}

Genes correlating with Alzheimer's disease (AD) susceptibility converge on immunoregulation, particularly those expressed in microglia. ${ }^{1}$ As the major immune cell in the brain, microglia play a myriad of roles and are connected with AD progression through their ability to phagocytose amyloid- $\beta .^{2}$ One of the key genes linked to AD susceptibility is the glycan-binding protein, CD33. ${ }^{3}$ The link between CD33 and AD susceptibility was identified through genomewide association studies, where a rare allele of CD33 is $A D$ protective. ${ }^{4,5}$ This rare allele undergoes alternative mRNA splicing ${ }^{6}$ leading to reduced expression of a long isoform of CD33 (CD33M; M=Major isoform), and increased expression of a short isoform (CD33m; m=minor isoform). Understanding the individual roles of these two CD33 isoforms has been challenging since human cells express a mixture of these two CD33 isoforms. Moreover, murine CD33 has several highly diverget features and does not undergo alternative mRNA splicing. ${ }^{7}$ Nevertheless, significant progress has recently been made and supports a role for CD33M in repressing phagocytosis, while distinct roles for CD33m have been also identified recently. ${ }^{7-10}$

Therapeutic targeting of proteins implicated in AD susceptibility has become an attractive approach. For example, antibodies targeting TREM2 have undergone rigorous evaluation in mouse models, ${ }^{11-13}$ and are now under clinical evaluation (NCT03635047). These antagonistic anti-TREM2 antibodies stimulate microglial cell proliferation and phagocytosis as a key means of skewing microglia into a disease protective phenotype. ${ }^{13}$ TREM2 is proposed to be negatively regulated by $C D 33,{ }^{14,15}$ and antibodies targeting CD33 - which have a long history in the clinic for the treatment of leukemia ${ }^{16}$ - are being assessed as means of modulating microglia in a clinical trial (NCT03822208). Anti-CD33 antibodies deplete CD33M from the surface, ${ }^{17-20}$ which is where it needs to be in order to repress phagocytosis. ${ }^{8}$ However, as murine CD33 does not serve as a good model for human CD33, ${ }^{7}$ in vivo pre-clinical testing of anti-CD33 antibodies have not been carried out. 
CD33 is a member of sialic acid-binding immunoglobulin-type lectin (Siglec) family of immunomodulatory receptors. ${ }^{21}$ CD33 binds to both $\alpha 2-3$ and $\alpha 2-6$ linked sialosides, with emerging evidence that binding may be enhanced by underlying sulfation. ${ }^{22,23}$ Engaging Siglecs with high-affinity and specific glycan ligands is an alternative approach compared to antibodies. ${ }^{24-}$ ${ }^{28}$ A high-affinity selective glycan ligand for targeting human CD33 was previously developed through dual modification of the 5- and 9-position of sialic acid with hydrophobic substituents. ${ }^{29}$ Recently, a high-resolution crystal structure of CD33 and this high-affinity CD33 ligand was determined, providing a basis for how the modifications exploit surrounding hydrophobic pockets. ${ }^{30}$ Moreover, this same study found that microparticles displaying this CD33 ligand increased the uptake of $A \beta$ peptide in human monocytic cells (THP1), although the mechanism by which this occurred was not elucidated. Regardless, these results suggest that targeting CD33 through its glycan-binding domain has the potential to modulate microglial phagocytosis.

Here, we developed and implemented liposomes displaying the high-affinity CD33 ligand (CD33L) for engaging CD33 and show that CD33L liposomes enhance phagocytosis in a CD33dependent manner. CD33L liposomes were formulated with two different fluorophores - one for monitoring CD33-engagement and a $\mathrm{pH}$-sensitive fluorophore for monitoring cellular internalization - that led to the discovery that the ability of CD33L liposomes to enhance phagocytosis strongly correlates with CD33 and liposome internalization within the cells. Moreover, CD33L liposomes enhance phagocytosis of primary mouse microglia expressing human CD33 following an intracerebroventricular injection. These results demonstrate the pharmacological potential of modulating immune cell function in the brain through targeting an immunomodulatory glycan-binding protein. 


\section{Results and Discussion}

Targeting CD33 with CD33L liposomes. A bifunctionally-modified version of Neu5Aca2$6 \mathrm{Gal} \beta 1-4 \mathrm{Glc}\left(\alpha 2-3\right.$ sialyl-lactose) 1 that has both selectivity ${ }^{29}$ and increased affinity $\left(K_{d}=87 \mu \mathrm{M}\right)^{22}$ for CD33 was used in these studies. For incorporation into liposomal nanoparticles, this synthetic CD33 ligand (CD33L) was conjugated to peggylated distearoylphosphatidylethanolamine (PEGDSPE) to form CD33L-PEG-DSPE 3 (Fig. 1a). For monitoring binding and internalization of liposomes to CD33-expressing cells, two fluorophores - AlexaFluor647 (AF647) and pH-sensitive rhodamine (pHrodo), respectively - were also conjugated to lipid to form AF647-PEG-DSPE 7 (Fig. 1b) and pHrodo-PEG-DSPE 6 (Fig. 1c), respectively. With these lipid conjugates in hand,

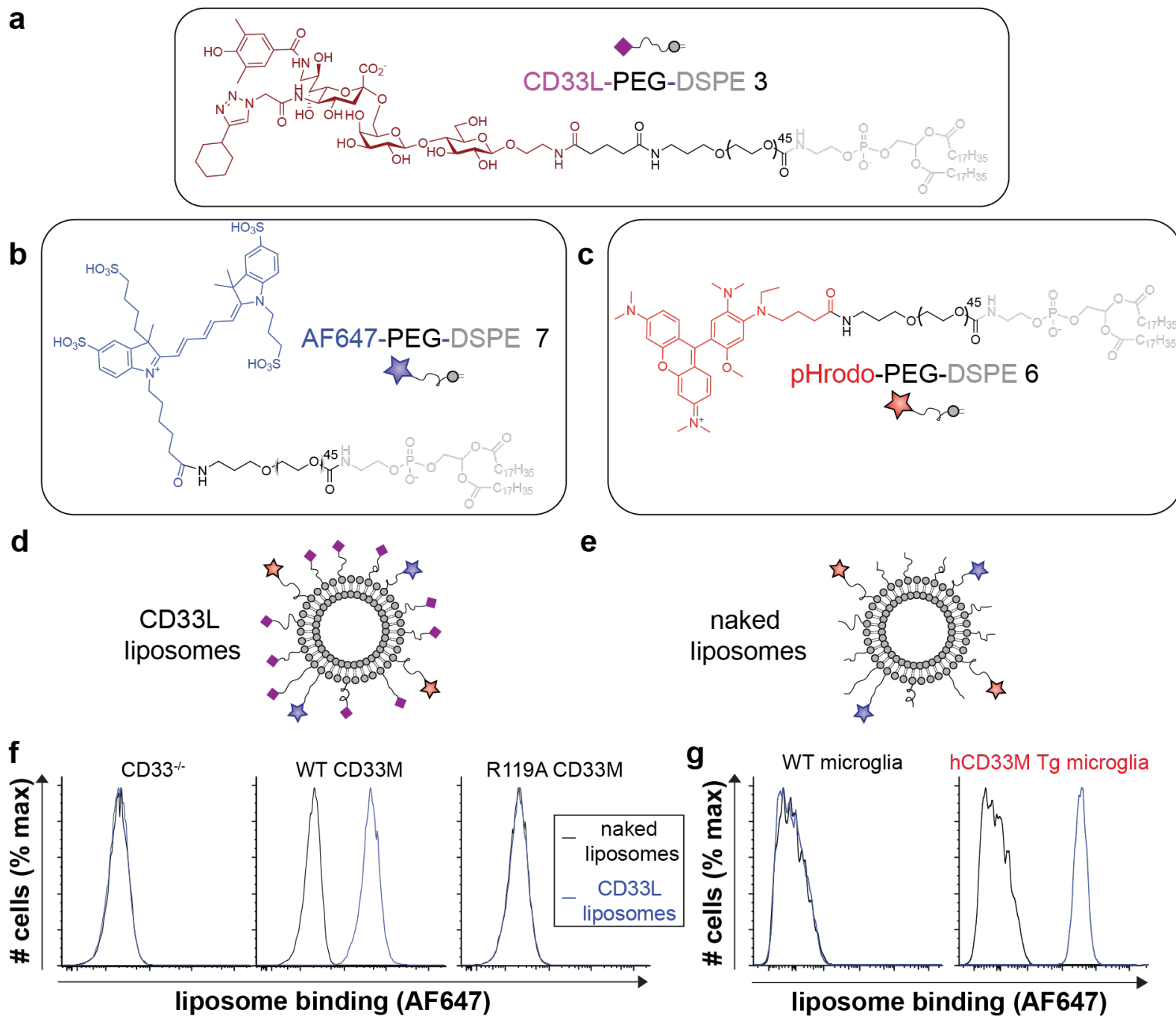


Fig. 1. Fluorophore conjugated CD33L liposome formulation for engaging CD33 on cells. (a-c) Chemical structures of CD33L-PEG-DSPE 3 (a), AF647-PEG-DSPE 7 (b), and pHrodoPEG-DSPE 6 (c). (d,e) Cartoon diagrams of CD33L (d) and naked (e) liposomes. (f) Binding of naked (black) and CD33L liposomes (blue) to CD33 ${ }^{-/}$(middle panel), and CD33-l- U937 cells transduced with WT CD33M (middle panel) or R119A CD33M (right panel). (g) Binding of naked (black) and CD33L liposomes (blue) to primary microglia isolated from WT $\left(\mathrm{Cx}_{3} \mathrm{Cre}^{\mathrm{Cre}-{ }^{+}+} h C D 33 \mathrm{M}^{-}\right.$ ${ }^{-}$) or hCD33M transgenic $\left(\mathrm{Tg} ; \mathrm{Cx}_{3} \mathrm{Cre}^{\mathrm{Cre}-/+} h C D 33 \mathrm{M}^{-/+}\right)$mice. Binding of liposomes to cells was measured by total AF647 signal by flow cytometry.

liposomal nanoparticles were formulated to contain $0.1 \mathrm{~mol} \%$ AF647-PEG-DSPE 7, $0.1 \mathrm{~mol} \%$ pHrodo-PEG-DSPE 6, and with or without 3 mol\% CD33L-PEG-DSPE 3 to make naked liposomes (Fig. 1d) and CD33L liposomes (Fig. 1e), respectively. The liposome formulation was modeled on an FDA-approved formulation that is well tolerated in vivo. ${ }^{31-33}$ Specifically, the base formulation of these liposomes contained a total of $5 \mathrm{~mol} \%$ PEG-DSPE, along with 57\% distearoylphosphatidylcholine, $38 \%$ cholesterol, and were extruded to $120+/-20 \mathrm{~nm}$ in size as determined by dynamic light scattering.

Liposomes were initially tested for binding to U937 cells by flow cytometry using the AF647 signal as a measure of total binding. CD33L liposomes showed robust binding to WT U937 cells, with no binding to CD33-/- U937 cells or CD33-/- U937 cells transduced with an R119A mutant of CD33 (Fig. 1f). Likewise, CD33L liposomes bound strongly to primary mouse microglia from human CD33M transgenic mice but not WT mouse microglia (Fig. 1g). These results demonstrate that CD33L liposomes specifically engage hCD33M on the cell surface of immune cells, which is in line with previous observations using a multivalent display of this ligand. ${ }^{29,32}$

CD33L liposomes increase phagocytosis in a CD33-dependent manner. We examined the effect of engaging CD33 with CD33L liposomes on phagocytosis by incubating cells with liposomes, washing the cells, and monitoring phagocytosis using fluorescent carboxylatemodified polystyrene beads in conjunction with flow cytometry. Initial experiments with the standard formulation (3.3 mol \% CD33L liposomes) significantly enhanced phagocytosis in WT but not CD33 ${ }^{-/-}$U937 cells, therefore, a series of CD33L liposomes were prepared with decreasing 
amounts of CD33L (Fig. 2a,b). Effects on phagocytosis were lost at CD33L densities lower than $3.3 \mathrm{~mol} \%$. Therefore, we moved forward with $3.3 \mathrm{~mol} \%$ CD33L density for further optimizations. Varying the concentration of CD33L liposomes, we find that enhanced phagocytosis was strongly correlated with the concentration of CD33L liposomes used and, notably, the highest concentration increased phagocytosis of WT U937 cells to nearly the level of CD33 ${ }^{-/-}$cells (Fig. 2c,d). As a final optimization, $3.3 \mathrm{~mol} \%$ CD33L liposomes at a concentration of $100 \mu \mathrm{M}$ were incubated for different amounts of time, with results indicating that phagocytosis increased for up to $2 \mathrm{hr}$ but no further increase was observed beyond $2 \mathrm{hr}$ (Fig. 2e,f). This data indicates that engagement of CD33 by CD33L liposomes increases phagocytosis in U937 cells in a manner that is dependent on CD33L density, liposome concentration, and time with which the liposomes are incubated with cells.

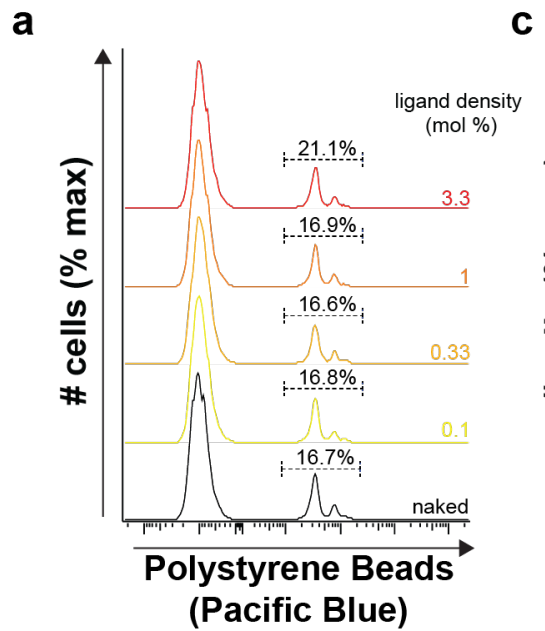

b

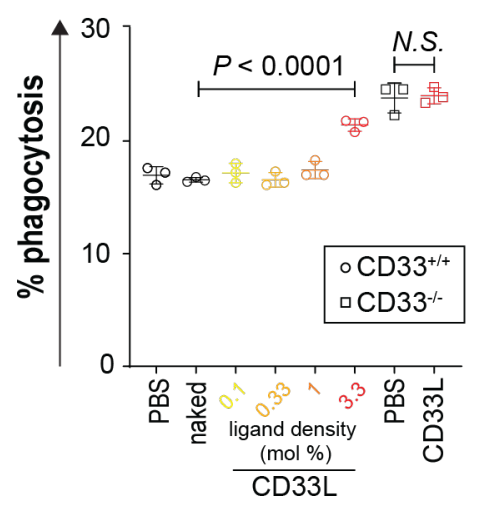

C

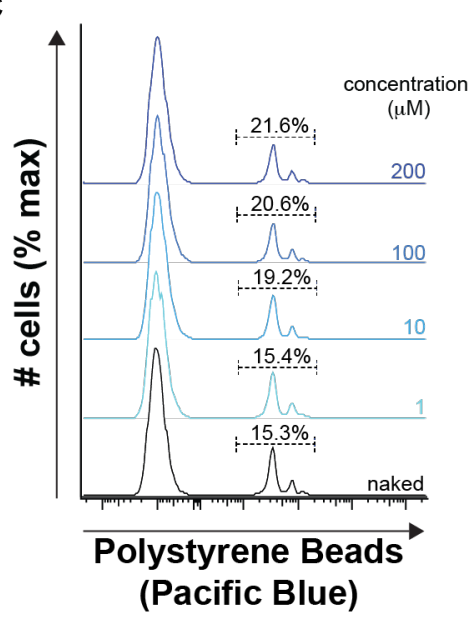

d

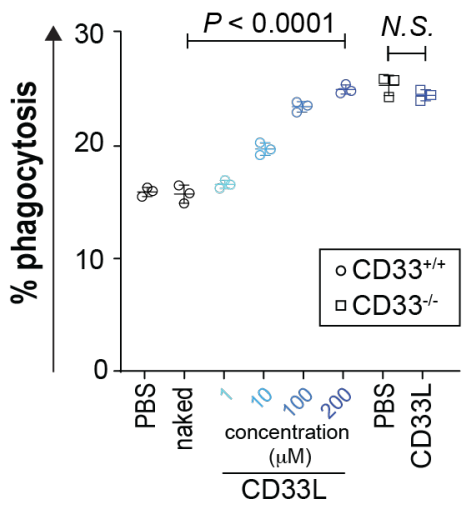

e

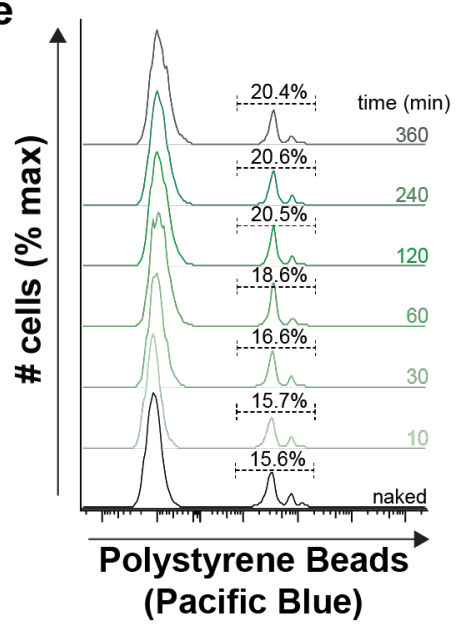

f

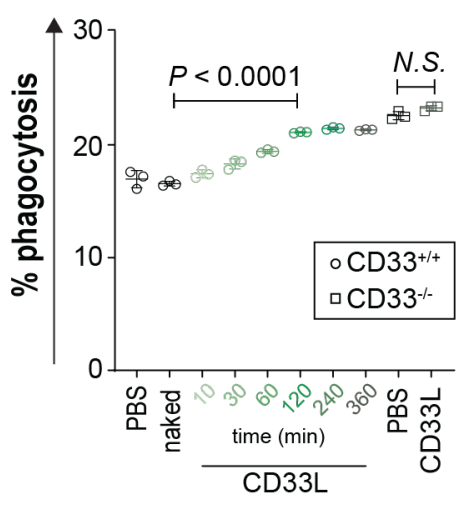


Fig. 2. CD33L liposomes increase phagocytosis of polystyrene beads in U937 cells. (a,b) Liposomal nanoparticles were formulated at different CD33L densities and tested for their ability to modulate phagocytosis. (c,d) The most efficient formulation (3.33 mol \% CD33L) was selected and tested at different liposomal concentrations. (e,f) Liposomal concertation of $100 \mu \mathrm{M}$ was selected and incubated with cells for various times before examining phagocytosis. Phagocytosis was measured by flow cytometry $(\mathbf{a}, \mathbf{c}, \mathbf{e})$ and the percentage of phagocytic cells was quantified $(\mathbf{b}, \mathbf{d}, \mathbf{f})$. Statistical significance was calculated using a one-way ANOVA with Dunnett's test.

Recently, we and others have demonstrated that the CD33 long isoform (CD33M) represses microglial phagocytosis, which is consistent with CD33M acting as an immunoinhibitory receptor. ${ }^{7-10}$ These results suggest that engaging CD33 with CD33L liposomes reverses this inhibitory effect. This is conceptually similar to results observed for another member of the Siglec family, CD22, where anti-CD22 antibodies enhance microglial phagocytosis. ${ }^{34}$

CD33L liposomes deplete cell surface CD33. As Siglecs are endocytic receptors, ${ }^{35}$ we investigated whether CD33L liposomes induce internalization of CD33. Internalization was assessed by depletion of CD33 from the cell surface using cell surface staining with an anti-CD33 antibody (clone HIM3-4) ${ }^{36}$ that does not bind the glycan-binding domain of CD33. In parallel, internalization of the liposomes was assessed using the fluorescent signal from pHrodo incorporated into the liposomes and comparing it to the fluorescent signal from AF647 that represents total liposome binding. For liposomes where the CD33L density was varied, $3.3 \mathrm{~mol}$ $\%$ CD33L induced the most significant depletion of CD33 from the cell surface (50\% depletion), liposome internalization, and liposome binding (Fig. 3a-f). Liposomes bearing $1 \mathrm{~mol} \%$ CD33L produced a modest depletion of cell surface CD33 levels as well as CD33 internalization, yet did not increase phagocytosis (Fig. 2a,b). Examining the concentration- (Fig. $3 g-\mathbf{I}$ ) and timedependent (Fig. 3m-r) effects of CD33L liposomes on internalization revealed that internalization of both CD33 and the CD33L liposomes strongly correlate with enhanced phagocytosis. Specifically, $200 \mu \mathrm{M}$ liposomes led to the greatest internalization of CD33 and liposomes, while internalization plateaued at $2 \mathrm{hr}$. 
a

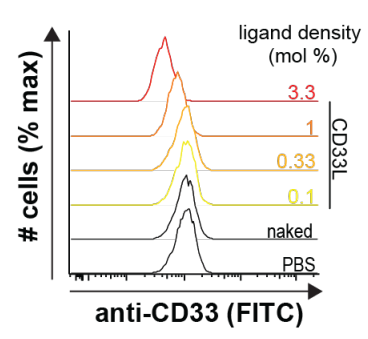

b

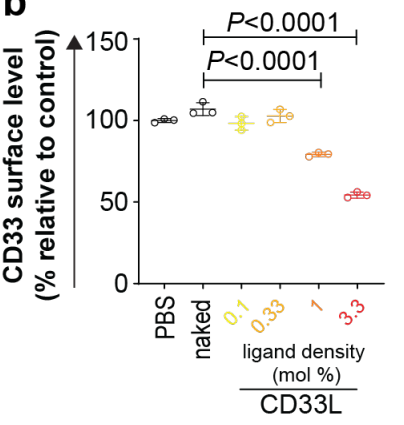

g

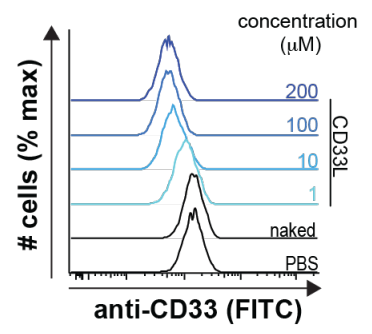

h

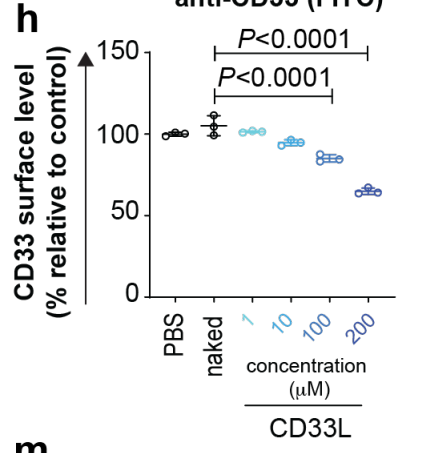

m

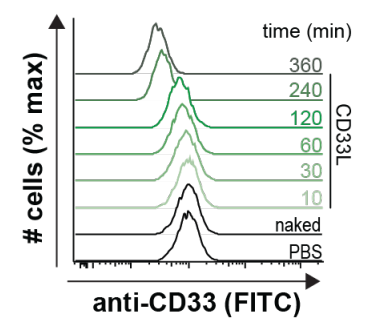

n

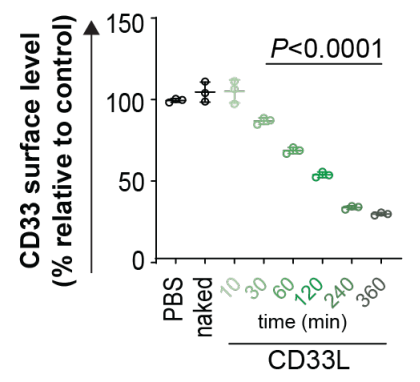

C
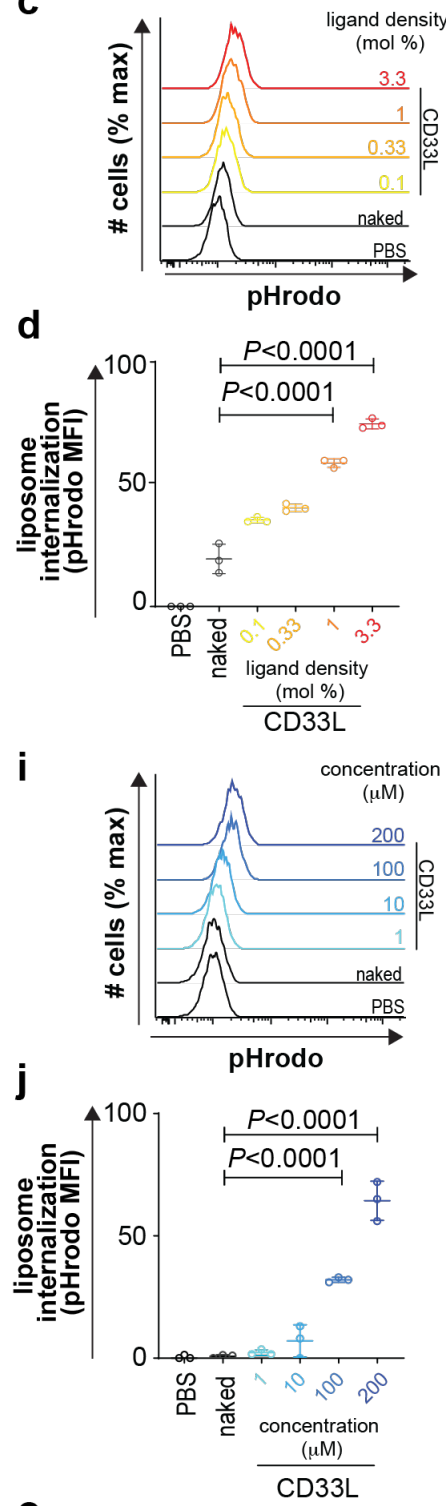

0
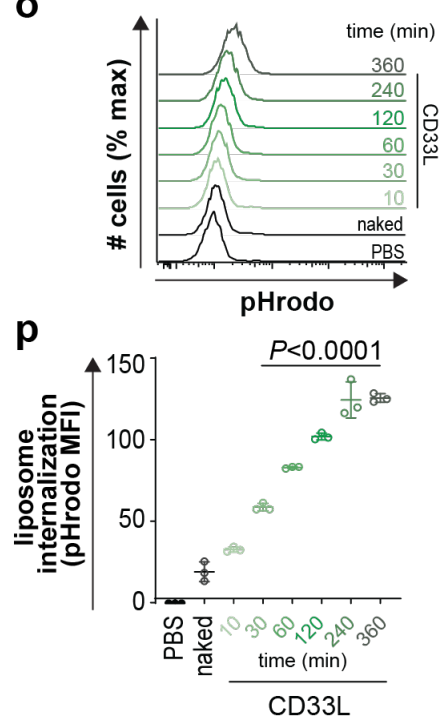
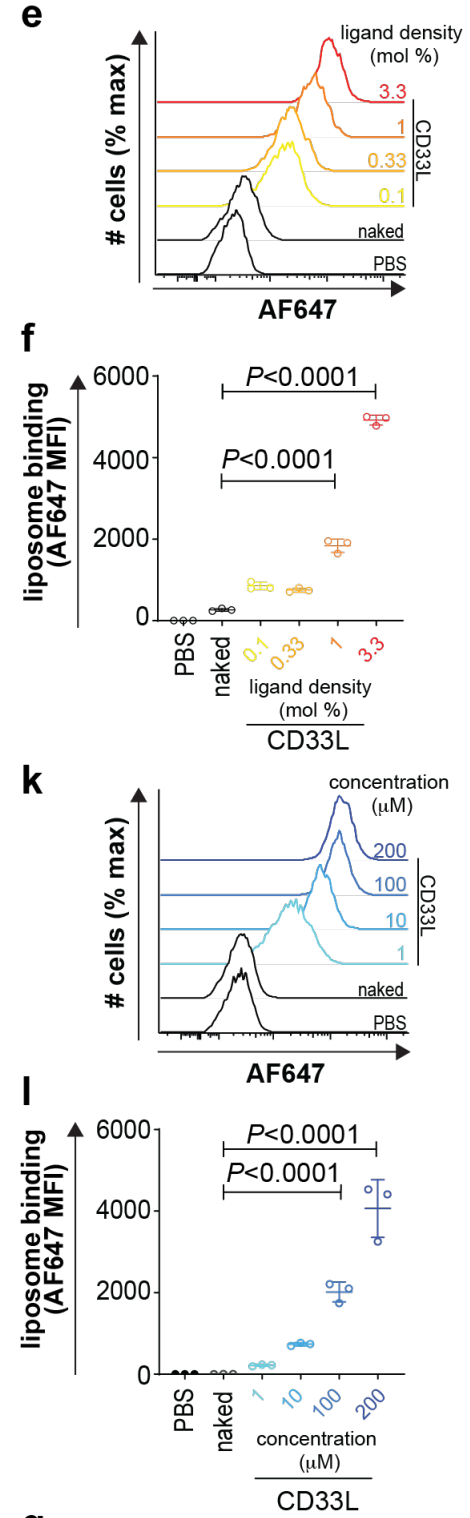

$\mathbf{q}$
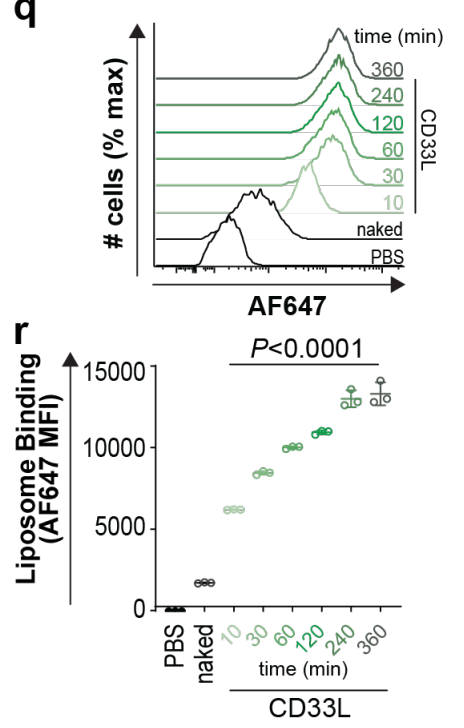
Fig. 3. CD33L liposomes induce internalization of CD33. (a-f) CD33L liposomes formulated with different CD33L densities, containing pHrodo and Alexa 647, were incubated with WT U937 cells. (g-I) CD33L liposomes formulated with 3.3\% CD33L were incubated with WT U937 cells at different concentrations. (m-r) CD33L liposomes were formulated with $3.3 \%$ CD33L and incubated with $\mathrm{U} 937$ cells at $100 \mu \mathrm{M}$ for different amounts of time. Cell surface CD33M cell surface staining (a,b,g,h,m,n), internalized liposomes (c, d, $\mathbf{i}, \mathbf{j}, \mathbf{o}, \mathbf{p})$, and total liposome binding (e,f,k,l, $\mathbf{q}, \mathbf{r})$ measured by flow cytometry. Raw flow cytometry plots $(\mathbf{a}, \mathbf{c}, \mathbf{e}, \mathbf{g}, \mathbf{i}, \mathbf{k}, \mathbf{m}, \mathbf{0}, \mathbf{q})$ and summary plots of the MFI values (b,d,f,h,j,I,n,p,r) are colour coded for the conditions used. Assays were replicated three times and statistical significance was calculated based on a one-way ANOVA with Dunnett's test.

We also examined CD33 and liposome internalization by confocal microscopy. Following incubation of U937 cells with CD33L liposomes, a 46\% decrease in cell surface CD33 was observed (Supplementary Figure S1a,b), which are highly consistent with the flow cytometry results. In these images, pHrodo and AF647 signal from the liposomes were also readily apparent in most CD33-expressing cells, where this signal was largely absent in cells incubated with naked liposomes. Using antibody HIM3-4 to track the CD33 inside the cell was not successful, therefore, a C-terminal 3xFLAG tagged version of CD33 was expressed in CD33 $3^{-/-}$U937 cells. This approach enabled fluorescence tracking of CD33, using an anti-FLAG antibody, where a strong signal was observed around the pHrodo and AF647 signal (Supplementary Figure S1c). These results strongly suggest that CD33 is internalized along with the CD33L liposomes.

Previous work using $5 \mu \mathrm{M}$ beads displaying this same ligand also induced an increase in phagocytosis, but due to the very large size of these beads it is unknown if they can be internalized and whether depletion of cell surface CD33 was at play. ${ }^{30}$ Our results demonstrate that internalization of CD33 closely correlates with increases in phagocytosis induced by CD33L liposomes. Interestingly, total CD33 engagement did not necessarily correlate with internalization and, consequently, increased phagocytosis. For example. CD33L liposomes bearing less than $3.3 \mathrm{~mol} \%$ CD33L still bound to CD33-expressing cells as evidenced by the AF647 signal (Fig. 3e), but did not deplete CD33 from the cell surface (Fig. 3a) or increase phagocytosis (Fig. 2a). Contrasting with this result, $10 \mu \mathrm{M}$ of liposomes (with the optimal $3.3 \mathrm{~mol} \% \mathrm{CD} 33 \mathrm{~L}$ ), had a similar 
overall ability to bind to cells (Fig. $\mathbf{3 k}$ ) but did deplete cell surface CD33 levels (Fig. $\mathbf{3 g}$ ) and increase phagocytosis (Fig. 2c). Therefore, the degree of crosslinking of CD33 on the cell surface, which is dictated by both the density and affinity of the CD33L, is the most critical element dictating internalization of CD33.

Role of glycan-binding and signaling motifs on phagocytosis. Binding of CD33 to its glycan ligands and control of immune cell signaling is dependent on a key salt bridge formed between Arg119 within its $\mathrm{N}$-terminal $\mathrm{V}$-set domain ${ }^{30}$ and signaling motifs within its cytoplasmic tail at Tyr340 (ITIM) and Tyr358 (ITIM-like) $)^{37}$, respectively. CD33-- U937 cells were previously established with WT CD33, R119A CD33, Y340A CD33, and Y358A CD33 re-introduced into these cells through lentiviral transduction. ${ }^{8}$ Here, these mutants were used to interrogate the roles of these key residues in the enhancement of phagocytosis by CD33L liposomes. Cell lines were treated with $100 \mu \mathrm{M}$ CD33L liposomes (3.3 mol\% CD33L) for $60 \mathrm{~min}$ before carrying out a phagocytosis assay with polystyrene beads. Compared to CD33-- cells (Fig 4a,b), cells reconstituted with WT CD33 showed enhanced phagocytosis by the treatment of CD33L liposomes (Fig. 4c,d). In both the R119A (Fig. 4e,f) and Y340A (Fig. 4g,h) mutant, the phagocytosis-enhancing effect of CD33L liposomes was lost. CD33L liposomes were still capable of enhanced phagocytosis in cells expressing the Y358A mutant (Fig. 4i,j). Previously, we showed that Y340 was critical for the ability of CD33 to repress phagocytosis, and others have also shown $\mathrm{Y} 340$ is required for antibody-mediated endocytosis of CD33. ${ }^{19,20}$ Consistent with these previous results, we find that the Y340A CD33 mutant internalizes significantly less as compared to WT CD33 in response to CD33L liposomes, whereas the Y358A CD33 mutant had no defect in internalization (Fig. 4K). 

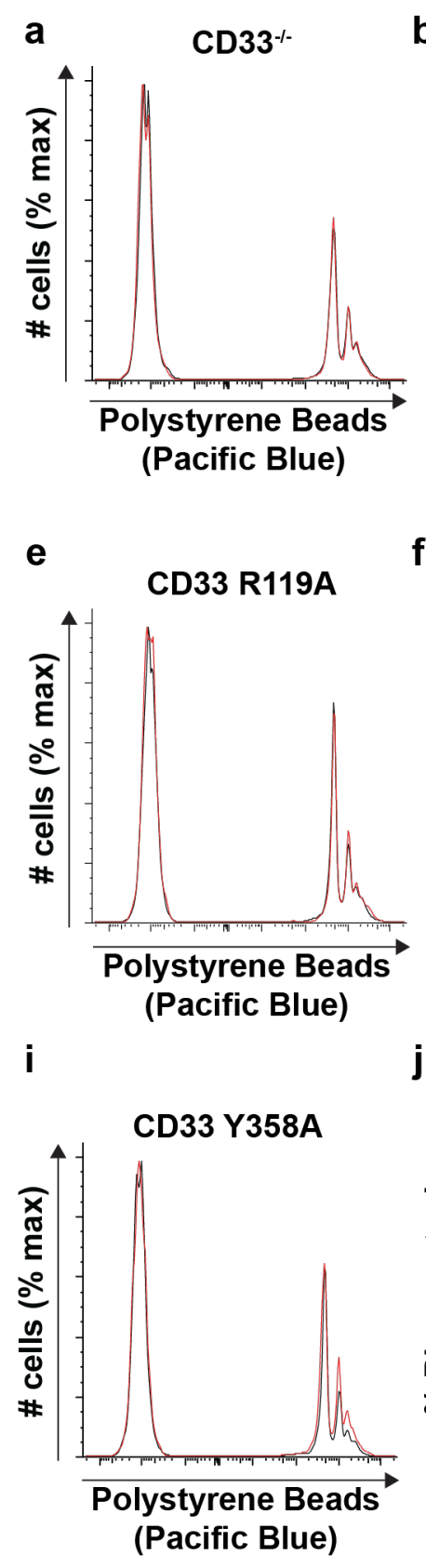
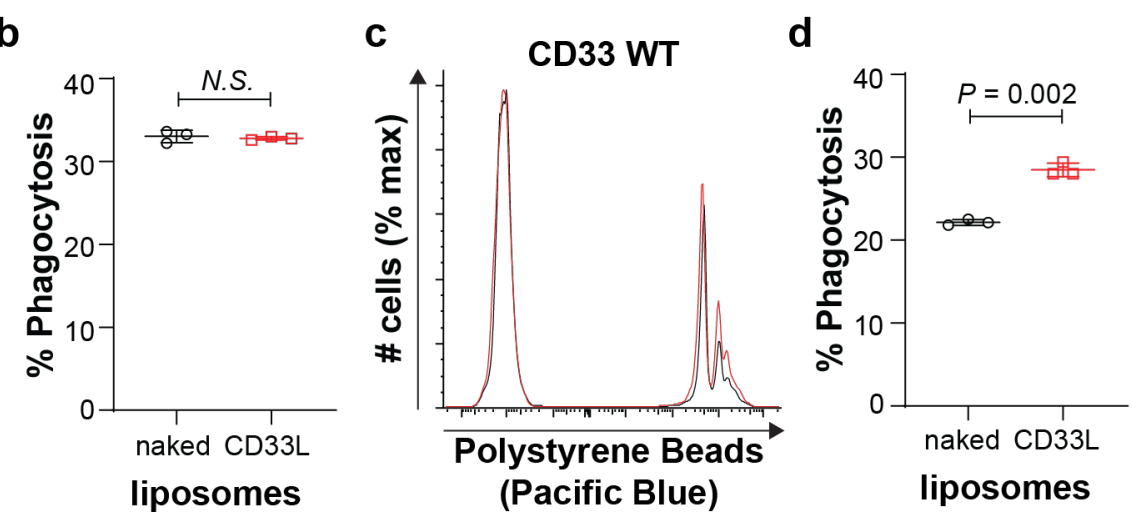

f
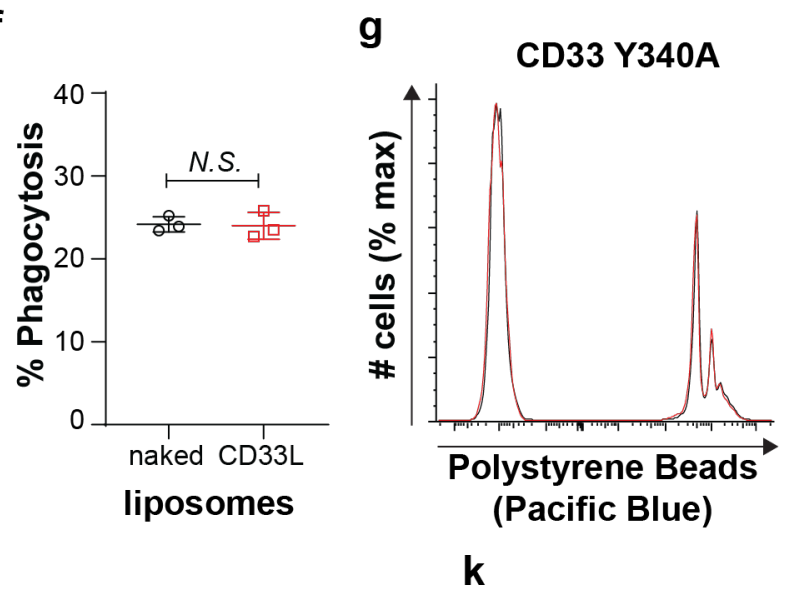

h

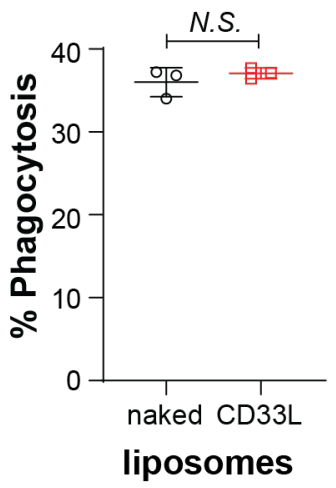

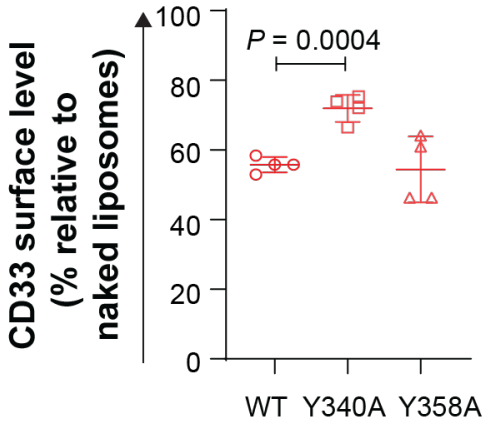

liposomes

Fig. 4. Increased phagocytosis by CD33L liposomes is dependent on the glycan-binding and signaling abilities of CD33. Naked (black) or CD33L (red) liposomes were incubated with CD33 $^{-/-}$U937 cells transduced with $(\mathbf{a}, \mathbf{b})$ empty vector, (c,d) WT CD33M, (e,f) R119A CD33M, $(\mathbf{g}, \mathbf{h})$ Y340A CD33M, (i,j) and Y358A CD33M prior to performing phagocytosis assay. Raw flow cytometry plots $(\mathbf{a}, \mathbf{c}, \mathbf{e}, \mathbf{g}, \mathbf{i})$ and summary plots of the MFI values $(\mathbf{b}, \mathbf{d}, \mathbf{f}, \mathbf{h}, \mathbf{j})$ are shown for each cell type. (k) CD33M internalization following incubation with naked liposomes or CD33L liposomes was examined on WT and CD33-- U937 cells transduced with Y340A or Y358A CD33M mutant. Assays were replicated three times and statistical significance was calculated based on aneway ANOVA with Dunnett's test. 
These results establish that the ability of CD33 to bind its glycan ligands, repress signaling, and internalize in response to CD33L liposomes are essential for the ability of CD33L liposomes to enhance phagocytosis. Recently, we showed that the ITIM of CD33 is essential for its ability to repress phagocytosis, whereas the ITIM-like residue is dispensable. ${ }^{8}$ Our findings here demonstrate that crosslinking alone of CD33, without its ability to modulate immune cell signaling through the ITIM, is insufficient for CD33L liposomes to impact phagocytosis. Previous results demonstrating a requirement for the ITIM of CD33 in antibody-mediated internalization ${ }^{20}$ are in agreement with our data. The optimized formulation of CD33L liposomes bares somewhere on the order of $1000-2000$ molecules of CD33L based on a $100 \mathrm{~nm}$ liposomes containing 84,000 lipids. This high multivalency along with our $120 \mathrm{~nm}$ liposomes falling within the range of efficient clathrin-mediated endocytosis gives these liposomes a strong ability to internalize surface receptors. ${ }^{38-41}$

Engaging CD33 in cis has the opposite impact on phagocytosis. Siglecs can be engaged by ligands on other cells (trans) or the same cell that the Siglec is expressed (cis) ${ }^{21}$. Results above with liposomes represent engagement with trans ligands. To test the impact of engagement with cis ligands, CD33L-PEG-DSPE 3 was inserted into cells (Supplementary Figure S2a). Successful insertion of CD33L into U937 cells was demonstrated by enhanced staining with CD33-Fc (Supplementary Figure S2b). Unlike trans engagement, cis engagement of CD33 with inserted CD33L led to a significant decrease in phagocytosis (Supplementary Figure S2c,d) in U937 cells expressing WT CD33. Insertion of the CD33L-PEG-DSPE 3 had no impact on phagocytosis within $\mathrm{CD}^{-/-}$U937 cells (Supplementary Figure S2e,f), nor did it impact phagocytosis in CD33 ${ }^{-/-}$U937 cells expressing R119A CD33 (Supplementary Figure S2g,h) or Y340A CD33 (Supplementary Figure S2i,j). These results are similar to recent work in which high-affinity glycan ligands of Siglec- 9 were inserted into cells, which induced a suppressive signal. ${ }^{42}$ The basis for how the engagement of CD33 with cis and trans ligands produce the 
opposite impact on phagocytosis is not completely understood, but results clearly indicate that the ITIM of CD33 is essential for both processes, which offers strong evidence for an immunomodulatory function of CD33.

\section{CD33L liposomes increase phagocytosis in primary microglia ex vivo. Results} demonstrating that CD33L liposomes enhance phagocytosis in cultured U937 cells motivated us to test if they also have a similar effect in microglia. As mouse CD33 is not functionally conserved with human CD33 (hCD33), we previously developed a transgenic mouse model in which $\mathrm{hCD} 33 \mathrm{M}$ is expressed in the microglial cell lineage and represses phagocytosis. ${ }^{7}$ We isolated microglia from WT and hCD33M transgenic mice and performed a competitive phagocytosis assay following incubation of cells with liposomes (Fig. 5a). Phagocytosis within the hCD33M ${ }^{+}$ and hCD33M- populations were deconvoluted using the gating scheme shown in Figure $\mathbf{5 b}$. The mixture of cells was incubated with either PBS (Fig. 5c,d), naked liposomes (Fig. 5,e,f), or CD33L liposomes (Fig. 5g,h). hCD33M transgenic microglia showed a decrease in phagocytosis compared to WT microglia in conditions where cells were treated with PBS and naked liposomes, which is anticipated based on the ability of hCD33M to repress phagocytosis in these transgenic mice $^{7,8}$. Pre-treatment with CD33L liposome increased phagocytosis in the CD33M microglia (Fig. 5i), such that no statistically significant difference was observed in phagocytosis between the WT and $\mathrm{hCD} 33 \mathrm{M}$ transgenic microglia. These results demonstrate that CD33L liposomes can enhance microglial cell phagocytosis in a CD33-dependent manner.

In vivo administration of CD33L liposomes enhanced phagocytosis in microglia. An advantage of our liposome formulation is that it is based on an FDA-approved formulation and is well-tolerated in vivo. ${ }^{32}$ However, this formulation does not penetrate the blood-brain barrier. ${ }^{43}$ Therefore, to test whether CD33L liposomes can successfully stimulate microglial phagocytosis 
a

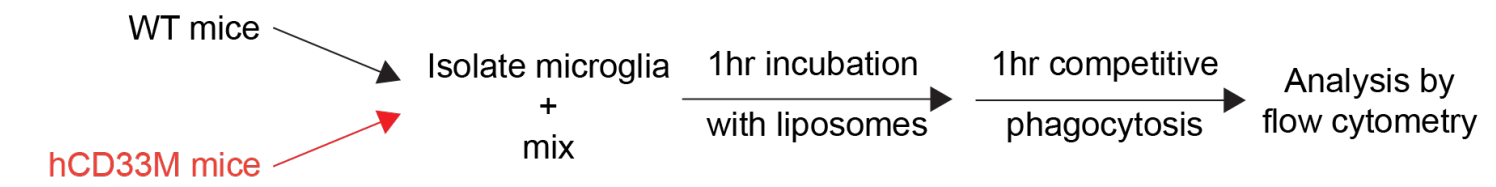

b
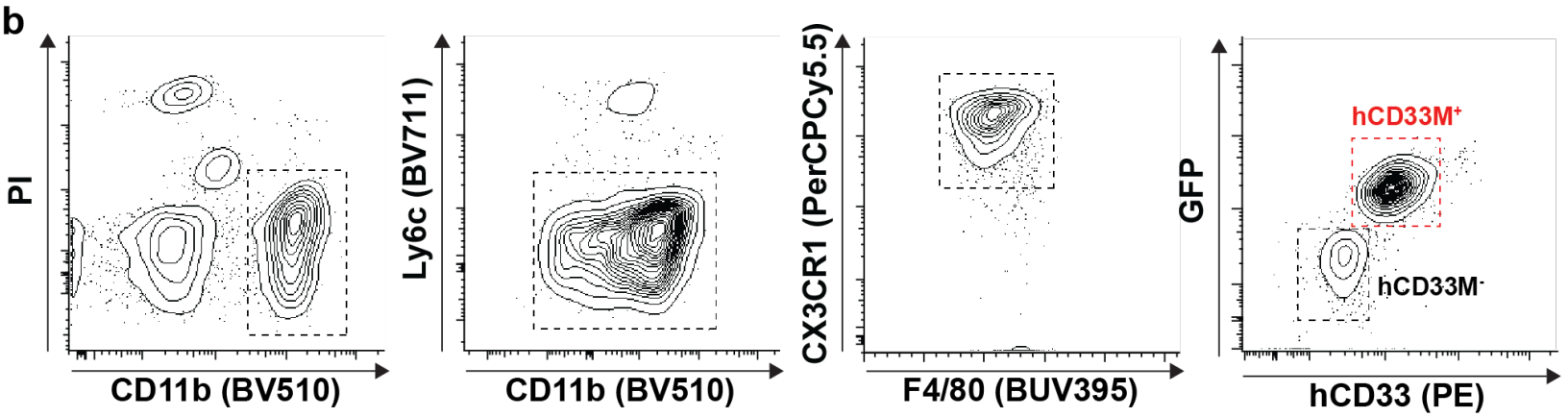

C

e

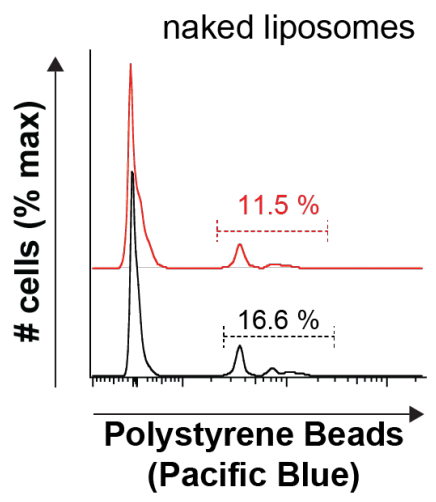

9

CD33L liposomes

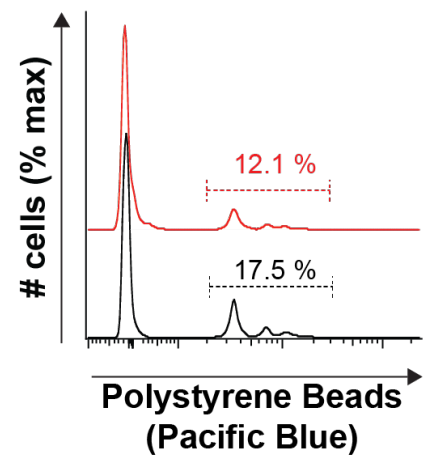

$\mathbf{f}$

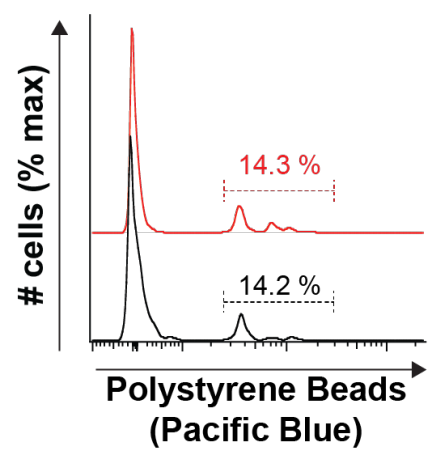

d

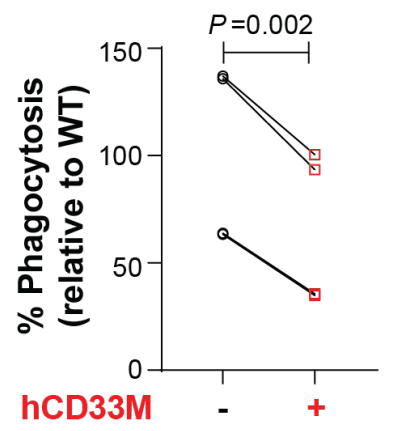

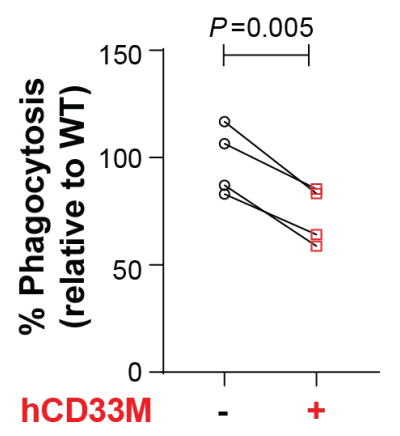

h

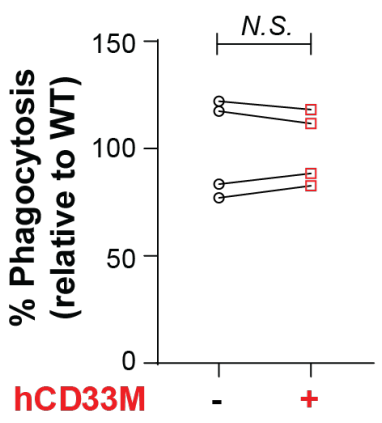

Fig. 5. CD33L liposomes enhance phagocytosis in hCD33M transgenic mouse microglia. (a) Schematic for a competitive phagocytosis assay with primary microglia taken from WT (black) and hCD33M transgenic (red) mice. (b) Gating strategy for the competitive phagocytosis assay. (c-h) Results of the competitive flow cytometry-based uptake of polystyrene microbeads. Isolated microglia were pretreated with PBS (c,d), naked liposome $(\mathbf{e}, \mathbf{f})$, or CD33L liposomes $(\mathbf{g}, \mathbf{h})$. Raw flow cytometric data $(\mathbf{c}, \mathbf{e , g})$ and the percentage of phagocytosis was quantified in each condition (d,f,h). (i) Absolute phagocytosis values in the hCD33M Tg microglia in the three conditions. Each point represents an independent experiment from different mice. N.S. represents no statistical significance based on a paired Student's t-test. 
in vivo, we administered liposomes via ICV injection. Briefly, WT and hCD33M transgenic mice were ICV injected with PBS, naked liposomes, or CD33L liposomes into the right ventricle, followed by a $4 \mathrm{hr}$ recovery prior to euthanization of mice. After euthanization brain samples were collected and microglia were isolated from each group (Fig. 6a). Initially, we performed a test with AF647 labeled naked and CD33L liposomes and examined AF647 fluorescence in isolated microglia, which demonstrated successful targeting of hCD33M-expressing cells selectively with CD33L liposomes (Fig. 6b,c). For assessing the impact on phagocytosis, WT or hCD33M Tg mice were carried out in parallel, and a competitive phagocytosis assay was used. In these studies, we assessed phagocytosis using the more physiologically-relevant aggregated fluorescent $A \beta_{1-42}$ and used conditions with and without Cytochalasin-D to control for non-specific sticking or uptake. Consistent with our results described above, hCD33M microglia mice injected with PBS (Fig. 6d,e) or naked liposome (Fig. 6f,g) showed repressed phagocytosis compared to their WT counterparts. On the other hand, CD33L liposome injected hCD33M mice (Fig. 6h,i) showed a similar level of phagocytosis as compared to WT mice under identical conditions. This data demonstrates that in vivo administration of CD33L liposome can modulate phagocytosis in microglia in a CD33-dependent manner.

In summary, our results demonstrate that CD33L liposomes can increase microglial phagocytosis both in vitro and in vivo. CD33L conjugated to microparticles produced a similar impact on phagocytosis in vitro, but due to their large size, these particles would not be suitable for in vivo use. ${ }^{44}$ We chose to use a biocompatible liposomal formulation ${ }^{45}$, which enabled CD33L density to be readily and systematically varied as well as be used in vivo. Indeed, CD33L liposomes co-displaying an antigen were successfully used to dampen allergic response in mice through exploiting the inhibitory function of CD33 on mast cells, however, CD33L liposomes in the absence of the allergen had no effect on the allergic response, consistent with the ability of 
$\mathbf{a}$
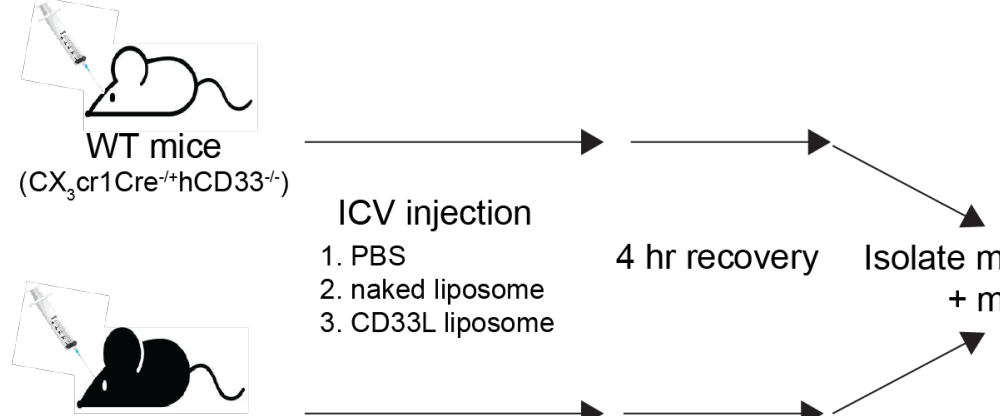

1. PBS

$4 \mathrm{hr}$ recovery Isolate microglia Competitive

2. naked liposome

3. CD33L liposome

hCD33M Tg Mice

$\left(\mathrm{CX}_{3} \mathrm{Cr} \mathrm{Cre}^{-/+} \mathrm{hCD} 33 \mathrm{M}^{-/+}\right)$
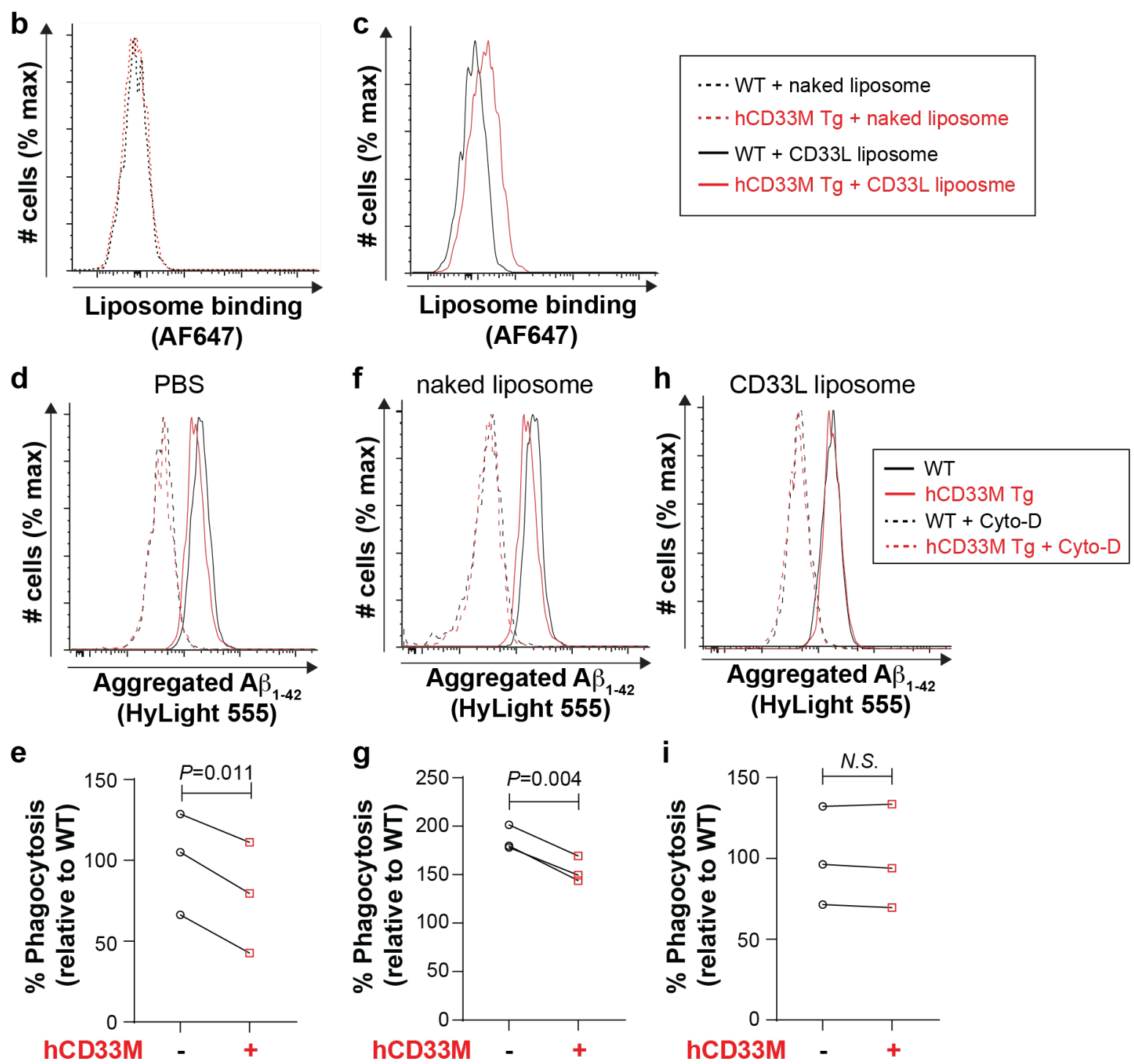

Analysis by

Phagocytosis flow cytometry

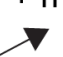

$$
\text { - hCD33M Tg + CD33L lipoosme }
$$

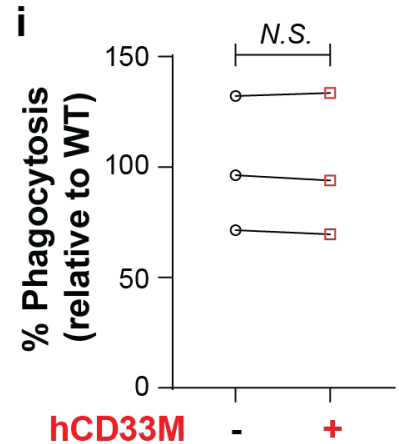


Fig. 6. Intracerebroventricular injection (ICV) of CD33L liposomes modulate phagocytosis of hCD33M microglia. (a) Schematic representation of the experimental plan, in which WT (black) or hCD33M transgenic mice (red) were administered PBS, naked liposomes, or CD33L liposomes, followed by isolation and mixing of microglia, and a competitive phagocytosis assay. (b,c) AF647 signal in microglia after injection of naked or CD33L liposomes. (d-i) Results of the competitive flow cytometry-based uptake of aggregated $A \beta_{1-42}$. WT (black) and hCD33M transgenic mice (red) in mice injected with PBS (d,e), naked liposome $(\mathbf{f}, \mathbf{g})$, or CD33L liposomes $(\mathbf{h}, \mathbf{i})$. Raw flow cytometric data $(\mathbf{d}, \mathbf{f}, \mathbf{h})$ and the percentage of phagocytosis was quantified in each condition $(\mathbf{e}, \mathbf{g}, \mathbf{i})$. Each point represents an independent experiment from different mice. N.S. represents no statistical significance based on a paired Student's t-test.

CD33 to antogonize Fc\&RI signaling, but only when the two receptors are co-ligated. ${ }^{32}$ Modulating the function of microglia in the brain is the next frontier in the treatment of neurodegenerative disease. Clinically used antibodies directly targeting $A \beta$ plaque accumulation have shown some promise but have ultimately not been successful. ${ }^{46-49}$ Thus, augmenting the phagocytic ability of immune cells in the brain may be a better approach. Antibody-based clinical trials are currently underway and while antibodies have long circulatory half-lives, they don't efficiently cross the blood-brain barrier. ${ }^{50,51}$ Multivalent engagement of Siglecs with glycan ligands offers several advantages over antibodies, including differences in cellular trafficking upon engagement and lower immunogenicity. ${ }^{24}$ CD33 has long been used as a target of leukemia for antibody-drug conjugates, due to the expression of CD33 in AML. ${ }^{16}$ The long-term safety associated with CD33directed antibody therapies is well established and results demonstrating that mice reconstituted with $\mathrm{CD}_{3} 3^{-/-}$human immune cells showed normal immune cell function provide further evidence that CD33-engaging therapies will be tolerated. ${ }^{52}$

\section{Methods}

Reagents and instrumentation. Reagents were purchased from commercial sources as noted and used without additional purification. NHS-pHrodo 4, anhydrous DMF, and $\mathrm{Et}_{3} \mathrm{~N}$ were purchased from Sigma-Aldrich, Canada. NHS-PEG-DSPE 2 and DSPE-PEG-NH 2 (2000) 5 were purchased from NOF America and Avanti, respectively. CD33L 1 was synthesized through the GlycoNet synthetic core. $\mathrm{CDCl}_{3}$ and $\mathrm{MeOD}_{4}$ were purchased from Deutero $\mathrm{GmbH}$. Other solvents 
(analytical and HPLC grade) and reagents were purchased from Aldrich and were used as received. Reactions were monitored by analytical TLC on silica gel 60-F254 (0.25 nm, Silicycle, QC, Canada). Developed TLC plates were visualized under UV lamp $(\lambda \max =254 \mathrm{~nm})$ and charred by heating plates that were dipped in ninhydrin solution in ethanol, and acidified anisaldehyde solution in ethanol. The reaction mixture was purified by silica gel column chromatography (230-400 mesh, Silicyle, Qc, Canada), Sephadex G-100 gel filtration chromatography using $\mathrm{CH}_{2} \mathrm{Cl}_{2} / \mathrm{MeOH}$ or $\mathrm{H}_{2} \mathrm{O}$ as the elution solvent. NMR experiments were conducted on a Varian 600, or $700 \mathrm{MHz}$ instruments in the Chemistry NMR Facility, University of Alberta. Chemical shifts are reported relative to the deuterated solvent peak or 3-(trimethylsilyl)propionic-2,2,3,3-d 4 acid sodium salt as an internal standard and are in parts per million (ppm). Coupling constants $(J)$ are reported in $\mathrm{Hz}$ and apparent multiplicities were described in standard abbreviations as singlet (s), doublet (d), doublet of doublets (dd), triplet (t), broad singlet (bs), or multiplet (m).

Synthesis of CD33L-PEG-DSPE 3: Chemical reaction scheme is presented in Supplementary Figure S3. To a solution of CD33L-NH $21(1.86 \mathrm{mg}, 1.9 \mu \mathrm{mol}, 1.0$ equiv) and NHS-activated DSPE-PEG 2 (5 mg, $1.66 \mu \mathrm{mol}, 1$ equiv) in anhydrous DMSO (150 $\mu \mathrm{L}, \sim 10 \mathrm{mM}$ ) was added diluted solution of $\mathrm{Et}_{3} \mathrm{~N}$ (1.5 equiv.) in anhydrous $\mathrm{DMSO}$ and $\mathrm{pH}$ of the solution was adjusted between 7.5-8 and the reaction mixture was incubated at room temperature for $6 \mathrm{~h}$. An aliquot of the reaction mixture was taken for TLC $\left(\mathrm{CHCl}_{3}: \mathrm{MeOH}: \mathrm{H}_{2} \mathrm{O}=\sim 75: 23: 2\right)$ analysis. Consumption of the NHS-activated PEG-DSPE and progress of conjugation was monitored by TLC in iodine chamber (to stain CD33L-PEG-DSPE 3 conjugate and NHS-PEG-DSPE 2), ninhydrin (to stain CD33L amine), and p-anisaldehyde (CD33L-PEG-DSPE 3) staining solution. The solvent was removed and the crude product was loaded to Sephadex G-100 gel filtration column using $\mathrm{H}_{2} \mathrm{O}$ and the crude product was purified using $\mathrm{H}_{2} \mathrm{O}$ as an eluent to afford CD33L-PEG-DSPE 3 as a white powder after lyophilization of fractions having the desired product. Yield: (5.81 mg, 90\%), 
coupling efficiency 70\%. ${ }^{1} \mathrm{H}$ NMR $\left(700 \mathrm{MHz} \mathrm{MeOD}_{4}\right): \delta 7.69(\mathrm{~s}, 1 \mathrm{H}), 7.84(\mathrm{~s}, 2 \mathrm{H})$, 5.23-5.20 (m, 1H), 5.09 (dd, $J=16.1,48.6 \mathrm{~Hz}, 2 \mathrm{H}), 4.40(\mathrm{dd}, J=3.6,12.6 \mathrm{~Hz}, 1 \mathrm{H}), 4.31(\mathrm{~d}, J=7.7 \mathrm{HZ}, 1 \mathrm{H})$, $4.29(\mathrm{~d}, J=7.7 \mathrm{HZ}, 1 \mathrm{H}), 4.19(\mathrm{~d}, J=6.3 \mathrm{HZ}, 1 \mathrm{H}), 4.17-4.15(\mathrm{~m}, 2 \mathrm{H}), 4.06-4.02(\mathrm{~m}, 1 \mathrm{H}), 3.97(\mathrm{t}, J$ $=5.6 \mathrm{~Hz}, 2 \mathrm{H}), 3.92-3.86(\mathrm{~m}, 5 \mathrm{H}), 3.82-3.80(\mathrm{~m}, 3 \mathrm{H}), 3.73(\mathrm{t}, J=7.7 \mathrm{HZ}, 1 \mathrm{H}), 3.70-3.62(\mathrm{~m}, 5 \mathrm{H})$, 3.64 (broad s, 161H), 3.59-3.51 (m, 12H), 3.48-3.46 (m, 1H), 3.42-3.40 (m, 4H), 3.34-3.32 (m, 3H), 3.31 (broad s, 44H), $3.24(\mathrm{t}, J=6.3 \mathrm{~Hz}, 2 \mathrm{H}), 3.04(\mathrm{t}, J=6.3 \mathrm{~Hz}, 1 \mathrm{H}), 2.34-2.29(\mathrm{~m}, 5 \mathrm{H}), 2.23$ (s, 6H), 2.22-2.18 (m, 4H), 2.42-2.22 (m, 2H), $1.89(\mathrm{t}, J=6.8 \mathrm{~Hz}, 1 \mathrm{H}), 1.84-1.72(\mathrm{~m}, 2 \mathrm{H}), 1.76-$ $1.73(\mathrm{~m}, 2 \mathrm{H}), 1.60-1.58(\mathrm{~m}, 4 \mathrm{H}), 1.44-1.42(\mathrm{~m}, 4 \mathrm{H}), 1.30$ (broad $\mathrm{s}, 64 \mathrm{H}), 0.89(\mathrm{~s}, 6 \mathrm{H})$; (Supplementary Figure S4).The MALDI-TOF-MS spectrum showed the average mass centered at $3.8 \mathrm{kDa}$ and expected average mass was $3.8 \mathrm{kDa}$ (Supplementary Figure S5). The coupling efficiency was determined through assigning aromatic protons signals at $7.69 \mathrm{ppm}(\mathrm{s}), 7.84 \mathrm{ppm}$ (s) of the phenyl moiety at C9 position of bifunctionally substituted Neu5Ac with terminal methyl groups at $0.89 \mathrm{ppm}(\mathrm{s})$ of the DSPE lipid.

Synthesis of pHrodo-PEG-DSPE 6. Chemical reaction scheme is presented in Supplementary Figure S6.To a solution of NHS-activated pHrodo 4 ( $1 \mathrm{mg}, 1.52 \mu \mathrm{mol}, 1.0$ equiv.) and $\mathrm{NH}_{2}$-PEGDSPE 5 (5.3 mg, $1.90 \mu \mathrm{mol}, 1.25$ equiv.) in anhydrous DMF (10 mM) was added diluted solution of base $\mathrm{Et}_{3} \mathrm{~N}$ in anhydrous to adjust $\mathrm{pH}$ of the reaction mixture between $7.5-8$ and the solution was stirred for about $2 \mathrm{~h}$ at room temperature under dark. Solvent was removed under reduced pressure and the crude was purified by flash silica gel chromatography using gradient elution (MeOH to $\mathrm{CH}_{2} \mathrm{Cl}_{2}$ 1:9 v/v). Yield: $(4.90 \mathrm{mg}, 88 \%$ ). Coupling efficiency of 6 with the DSPE-PEG scaffold was determined by ${ }^{1} \mathrm{H}$ NMR integration. ${ }^{1} \mathrm{H}$ NMR $\left(600 \mathrm{MHz}, \mathrm{CDCl}_{3}\right): \delta 7.54(\mathrm{~d}, J=9.6$ $\mathrm{Hz}, 2 \mathrm{H}), 7.45(\mathrm{~s}, 1 \mathrm{H}), 7.10(\mathrm{~s}, 1 \mathrm{H}), 7.01(\mathrm{~d}, J=1.8 \mathrm{~Hz}, 1 \mathrm{H}), 6.99(\mathrm{~d}, J=1.8 \mathrm{~Hz}, 1 \mathrm{H}), 6.80(\mathrm{~d}, J=$ $1.8 \mathrm{~Hz}, 1 \mathrm{H}), 6.67(\mathrm{~d}, J=28.2 \mathrm{~Hz}, 1 \mathrm{H}), 5.24-5.22(\mathrm{~m}, 1 \mathrm{H}), 4.42-4.22(\mathrm{~m}, 1 \mathrm{H}), 4.20-4.18(\mathrm{~m}, 2 \mathrm{H})$, 3.98-3.99 (m, 2H), 3.76-3.68 (m, 2H), 3.64 (broad s, 182H), 3.54-3.51 (m, 2H), 3.48-3.44 (m, 4H), 3.42-3.40 (m, 2H), $3.34(\mathrm{~s}, 6 \mathrm{H}), 2.70(\mathrm{~s}, 3 \mathrm{H}), 2.50(\mathrm{t}, J=6.0 \mathrm{~Hz}, 1 \mathrm{H}), 2.31-2.27(\mathrm{~m}, 3 \mathrm{H}), 2.05-$ 
$2.03(\mathrm{~m}, 2 \mathrm{H}), 1.93-1.91(\mathrm{~m}, 2 \mathrm{H}), 1.27$ (broad s, 64H), $1.15(\mathrm{t}, \mathrm{J}=6.6 \mathrm{~Hz}, 3 \mathrm{H}), 0.89(\mathrm{~s}, 6 \mathrm{H}), 0.85$ (s, 6H); (Supplementary Figure S7). The MALDI-TOF-MS spectrum showed the average mass centered at $3680 \mathrm{Da}$ and expected average mass was $3680 \mathrm{Da}$ (Supplementary Figure S8).

Synthesis of AF647-PEG-DSPE 7. Compound 7 was prepared by following a procedure described previously. ${ }^{32}$

Liposome Preparation. Commercially available lipids such as DSPC, Cholesterol, DSPE-PEG were purchased and 10,5 , and $4 \mathrm{mg} / \mathrm{mL}$ stock solutions were made respectively. In parallel stock solutions of pHrodo-PEG-DSPE 6 (1mg/mL), AF647-PEG-DSPE 7 (1mg/mL), CD33L-PEGDSPE $3(3.2 \mathrm{mg} / \mathrm{mL})$ were prepared. For naked liposomes $57,38,4.8,0.1$, and $0.1 \mathrm{~mole} \%$ of DSPC, Cholesterol, DSPE-PEG, pHrodo-PEG-DSPE 6, AF647-PEG-DSPE 7 were used respectively. Similar mol \% of DSPC, Cholesterol, pHrodo-PEG-DSPE 6, AF647-PEG-DSPE 7 were used for CD33L liposomes with variable concentration $(0.1,0.33,1$, and $3.33 \mathrm{~mol} \%)$ of CD33L-PEG-DSPE 3 keeping total PEG-DSPE concentration at 5\%. Briefly, DSPC, Cholesterol, DSPE-PEG were suspended in chloroform and an appropriate volume of each lipid solution in chloroform was transferred into a glass test tube to reach the desired mol \% of each lipid. The solvent was removed under nitrogen gas to form the lipid mixtures. Once all visible chloroform was removed and approximately $100 \mu \mathrm{L}$ of dimethyl sulfoxide (DMSO) was added to the test tube. CD33L-PEG-DSPE 3, pHrodo-PEG-DSPE 6, and 0.1\% of DSPE-PEG-A647 7 in DMSO were then added to the lipid mixture in appropriate proportions to reach the desired mol $\%$ of each CD33L. The samples were placed at $-80^{\circ} \mathrm{C}$ until completely frozen and excess DMSO was removed via lyophilization overnight and then the dried liposomes were stored at $-80^{\circ} \mathrm{C}$ until they were extruded.

Dried lipids were allowed to warm to room temperature and were then hydrated with 1.0 $\mathrm{mL}$ of phosphate-buffered saline $\mathrm{pH} 7.4$ (Gibco). The hydrated lipids were then sonicated in a cycle of 1 minute on, 4-5 minutes off until all lipids were uniformly suspended. The lipids were 
extruded with an $800 \mathrm{~nm}$ filter followed by $100 \mathrm{~nm}$ filters. The size of the liposomes was then verified by dynamic light scattering (Malvern PanalyticalZetasizer Nano S) to be approximately $120+-/-20 \mathrm{~nm}$. Liposomes were stored in at $4^{\circ} \mathrm{C}$.

Animal Studies. All mice were on a C57BL/6J genetic background. Transgenic mice expressing hCD33M in the Rosa26 locus were prepared according to previously published methodology. ${ }^{32}$ These mice are widely characterized and tested in our recently published studies. ${ }^{7,8}$ All animals used were maintained in an access-controlled barrier facility under specific-pathogen-free conditions. Studies were approved by the Health Sciences Animal Care and Use Committee of the University of Alberta (AUP00002885).

Cell lines. WT and CD33 $3^{-/-}$U937 used for these studies were generated as a part of another published study. ${ }^{8}$

Liposome binding studies. U937 cells were grown to a density of $\sim 1 \times 10^{6}$ cells $/ \mathrm{mL}$ in a T175 flask before the assay, harvested, centrifuged, resuspended in media, and 100,000 cells were added to a 96 -well U-bottom plate in $200 \mu \mathrm{L}$ of media. Cells were centrifuged at $300 \mathrm{rcf}$ for $5 \mathrm{~min}$ and the supernatant was discarded. The cell pellet was re-suspended in $50 \mu \mathrm{L}$ of fresh media and $50 \mu \mathrm{L}$ of media containing liposomes was added to it. The final concentration of liposomes was $100 \mu \mathrm{M}$ in each well. The CD33L concentration on the liposomes was $3.33 \mathrm{~mol} \%$ and the liposome size was $100 \mathrm{~nm}$. These suspensions were incubated for 60 minutes at $37^{\circ} \mathrm{C}$ and $4{ }^{\circ} \mathrm{C}$ (as a negative control). Following this incubation, $100 \mu \mathrm{L}$ of media was added to each sample and they were centrifuged at 300 rcf for $5 \mathrm{~min}$. After centrifugation, the supernatant was discarded, and the cell pellet was suspended in flow buffer and further analyzed by flow cytometry. All synthesized liposomes were fluorescently labeled with AF647, which allows us to determine the target specificity of synthesized liposomes to engage the CD33 receptor on the cell surface. The extent of this parameter was determined by assessing the median fluorescence intensity (MFI) of 
the AF647 fluorescent signal. In each case, cells without liposome treatment were kept to subtract non-specific signals from experimental values.

Optimization of liposome formulations and their effect on CD33 internalization. U937 cells were grown in a T175 flask and 100,000 cells were added to a 96-well U-bottom plate in $200 \mu \mathrm{L}$ of media. Cells were centrifuged at 300 rcf for $5 \mathrm{~min}$ and cell-pellet was re-suspended in $50 \mu \mathrm{L}$ of fresh media, and $50 \mu \mathrm{L}$ of media containing liposome was added to it. These suspensions were incubated for 120 minutes at $37^{\circ} \mathrm{C}$ and $4{ }^{\circ} \mathrm{C}$. To best optimize the assay we started with liposome containing different concentrations of ligand $(0.1,0.33,1$, and $3.33 \mathrm{~mol} \%)$. Furthermore, we varied liposome concentration $(1,10,100$, or $200 \mu \mathrm{M})$ to understand how this factor can influence internalization. Finally, we performed a time (10, 30, 60, 120 240, and 360 min) dependedinternalization assay with synthesized liposomes. Following the incubation period with the desired liposome, $150 \mu \mathrm{L}$ of flow buffer was added in each well, and samples were centrifuged at $300 \mathrm{rcf}$ for $5 \mathrm{~min}$. After centrifugation supernatant was discarded and the pellet was stained with $50 \mu \mathrm{L}$ FITC labeled HIM 3-4 antibody (1:100 dilution from a 1mg/mL stock solution) for 30 minutes at 4 ${ }^{\circ} \mathrm{C}$. Following this incubation $150 \mu \mathrm{L}$ of flow buffer was added to each sample and they were centrifuged at $300 \mathrm{rcf}$ for $5 \mathrm{~min}$. After centrifugation, the supernatant was discarded, and the cell pellet was suspended in flow buffer for further analysis by flow cytometry. The extent of decrease of CD33 from cell surface was determined by assessing the MFI of the fluorescent signal observed in FITC channel and using the following formula: Decrease of CD33 from the cell surface $(\%)=$ 100X (Cells treated with liposome at $37^{\circ} \mathrm{C}$ - Cell without liposome treatment $37^{\circ} \mathrm{C}$ ) $/$ (Cells treated with liposome at $4^{\circ} \mathrm{C}$ - Cell without liposome treatment $4^{\circ} \mathrm{C}$ )

The addition of pHrodo and AF647 fluorophores to liposomes allowed us to determine cellular internalization and binding capability of liposomes respectively. The extent of these two parameters was determined by assessing the MFI of the pHrodo (PE channel) and AF647 
fluorescent signal respectively. Each case cells without liposome treatment were kept to determine non-specific signals and treatment at $4^{\circ} \mathrm{C}$ was treated as a negative control.

Effect of liposome on cellular phagocytosis. U937 cells were grown in a T175 flask and 100,000 cells were added to a $96-$ well U-bottom plate in $200 \mu \mathrm{L}$ of media. Cells were centrifuged at $300 \mathrm{rcf}$ for $5 \mathrm{~min}$ and the supernatant was discarded. The cell pellet was re-suspended in 50 $\mu \mathrm{L}$ of fresh media and $50 \mu \mathrm{L}$ of media containing liposomes was added. The final concentration of liposomes was $100 \mu \mathrm{M}$ in each well. In parallel, cells were treated with naked liposome which does not contain CD33 ligand. These suspensions were incubated for 60 minutes at $37^{\circ} \mathrm{C}$ or $4^{\circ} \mathrm{C}$ (negative control). Following the incubation period, $100 \mu \mathrm{L}$ of media was added to each well, and samples were centrifuged at 300 rcf for 5 min. After centrifugation, the supernatant was discarded. The cell pellet was re-suspended in $50 \mu \mathrm{L}$ of fresh media and $50 \mu \mathrm{L}$ of fluorescent polystyrene beads (Thermo Fisher) for 30 minutes at $37^{\circ} \mathrm{C}$ or $4^{\circ} \mathrm{C}$. The final concentration of the polystyrene beads in each well was a 1:100 dilution from a commercially purchased stock solution. Following this incubation, $100 \mu \mathrm{L}$ of media was added to each sample and they were centrifuged at $300 \mathrm{rcf}$ for 5 minutes. After centrifugation, the supernatant was discarded, and the pellet was suspended in flow buffer for further flow cytometric analysis. The extent of phagocytosis was determined by assessing the percentage of cells taking up at least one bead.

CD33L-PEG-DSPE insertion into cells. CD33L-PEG-DSPE 3 or PEG-DSPE $(1 \mathrm{mM})$ stock solutions were made in DMSO. To initiate the assay, cells were centrifuged at $300 \mathrm{rcf}$ for 5 minutes and the media was discarded. The cells were resuspended in $50 \mu \mathrm{L}$ of PBS containing $10 \mu \mathrm{M}$ CD33L-PEG-DSPE 3 or PEG-DSPE (containing 1\% DMSO) and incubated for $1 \mathrm{hr}$ at $37^{\circ} \mathrm{C}$. Cells were centrifuged at $300 \mathrm{rcf}$ for $5 \mathrm{~min}$ and the supernatant was discarded. Cells were used in either a phagocytosis assay with polystyrene beads for 30 minutes at $37^{\circ} \mathrm{C}$ or stained with WT or R119A CD33-Fc according to a published procedure ${ }^{22}$. 
Treatment of primary mouse microglia with CD33L liposome. Adult mice (WT and hCD33M) were euthanized under $\mathrm{CO} 2$ and their brains were collected and kept in ice-cold RPMI media with 10\% FBS, $100 \mathrm{U} / \mathrm{mL}$ Penicillin, and $100 \mu \mathrm{g} / \mathrm{ml}$ Streptomycin. Isolated brains were homogenized by $5 \mathrm{ml}$ syringe plungers in media through $40 \mu \mathrm{m}$ corning filter units under sterile conditions. Homogenized samples were centrifuged at $500 \mathrm{~g}$ for $5 \mathrm{~min}$ and the pellet was treated with $3 \mathrm{ml}$ of red blood cell lysis buffer (150 mM NH4Cl, $9 \mathrm{mM} \mathrm{NaHCO} 3$, and $0.1 \mathrm{mM}$ EDTA). Following centrifugation at $300 \mathrm{rcf}$ for $5 \mathrm{~min}$, the pellet was dissolved in $3 \mathrm{ml}$ of $30 \%$ Percoll (Percoll PLUS, GE Healthcare) and carefully layered on top of $70 \%$ Percoll and immediately centrifuged (650 rcf for $20 \mathrm{~min}$ ). Immune cells were isolated from the border between the two layers, washed (300 rcf, $5 \mathrm{~min}$ ), and resuspended in media. Isolated microglia from WT and hCD33M mice were mixed and treated with naked liposome or CD33L liposome $(100 \mu \mathrm{M})$ containing $3.33 \%$ ligand for 120 $\min$ at $37^{\circ} \mathrm{C}$. After the incubation period $100 \mu \mathrm{L}$ fresh media was used to wash excess liposomes and cells were centrifuged at 300 rcf for 5 min. Pellet was collected and treated with $200 \mathrm{nM}$ aggregated $A \beta$ or polystyrene beads (1:200 dilution from commercial stock) at $37^{\circ} \mathrm{C}$ for $30 \mathrm{~min}$. Following this incubation, $100 \mu \mathrm{L}$ of media was added to each sample and they were centrifuged at $300 \mathrm{rcf}$ for 5 minutes. After centrifugation, the supernatant was discarded, and the pellet was treated with an antibody cocktail containing CD11b (APC/Cy7, clone M1/70, BioLegend), Ly-6G (BV605, clone 1A8, BioLegend), Ly-6C (BV711, clone HK 1.4, BioLegend), Cx3cr1 (PerCP/Cy5.5, clone SA011F11, BioLegend), and F4/80 (BUV395 clone T45-2342, BD Horizon), hCD33 (PE clone WM53 or BV421 clone WM53 BioLegend) at $4^{\circ} \mathrm{C}$ for $30 \mathrm{~min}$. After the antibody staining, 50 $\mu \mathrm{L}$ flow buffer was added to the cell pellet and centrifuged at 300 rcf for 5 minutes. After centrifugation cell pellet was re-suspended in flow buffer and further analyzed by flow cytometry. To inhibit phagocytosis, cells were pre-treated with $10 \mu \mathrm{M}$ Cytochalasin-D for $30 \mathrm{~min}$.

Effect of CD33L liposome in CD33 mutant U937 cell lines. WT and mutant U937 cells were grown in a T175 flask and 100,000 cells were added to a 96-well U-bottom plate in $200 \mu \mathrm{L}$ of 
media. Phagocytosis assay was performed with polystyrene beads following the method described earlier. The extent of phagocytosis was determined by assessing the percentage of cells taking up at least one bead. In a separate assay, we also tested depletion of cell surface CD33 by FITC-conjugated HIM 3-4 antibody following the method described earlier.

Cloning of the hCD33M-FLAG tag fusion protein into the RP172 vector. A 3xFLAG-tag (GATTATAAGGACGACGATGATAAGGATTACAAGGATGATGACGATAAGGACTATAAAGATG ACGACGACAAG), containing 5' Agel and 3' Aval restriction sites and a 3' stop codon, was synthesized as a gBlocks ${ }^{\circledR}$ Gene Fragment (Integrated DNA Technologies). PCR was used to amplify the sequence and it was cloned into the RP172 vector cut with Agel. The plasmid was transformed into NEB® Stable competent E. coli cells (New England Biolabs), and isolated plasmids were checked for proper orientation and quality of the FLAG-tag sequence through sanger sequencing. The gene encoding hCD33M, but lacking its natrual stop codon, was subsequently cloned into this vector using the 5' Sphl and 3' Agel restriction sites to form the hCD33M-FLAG-tag fusion protein. This was transformed into NEB® Stable competent E. coli cells, and isolated plasmids were checked for quality of the hCD33M-FLAG-tag sequence through sanger sequencing. Lentivirus production and transduction in CD33 ${ }^{-/-}$was carried out the same as a previously published protocol. ${ }^{8}$

Cell staining and confocal microscopy. Approximately 200,000 CD33 ${ }^{-/-}$U937 cells expressing hCD33M-3xFLAG protein were treated with liposomes (3.3 mol \% CD33L or naked) at a final concentration of $100 \mu \mathrm{M}$ for $45 \mathrm{~min}$. The cells were collected and centrifuged at 300 rcf for 5 minutes and the media was discarded. The cells were washed in PBS and centrifuged. The PBS was discarded, and the cells were resuspended in $100 \mu \mathrm{L}$ of ice cold $3 \%$ paraformaldehyde (PFA), followed by $15 \mathrm{~min}$ incubation at $4^{\circ} \mathrm{C}$. Cells were centrifuged at $1200 \mathrm{rcf}$ for $5 \mathrm{~min}$, PFA was discarded, and the cells were washed with PBS as described above. 
For surface staining of CD33, cells were blocked with $100 \mu \mathrm{L}$ of $5 \%$ goat serum for $10 \mathrm{~min}$, followed by incubation with AF488 conjugated anti-CD33 (clone HIM3-4; 1:100 dilution) for $1.5 \mathrm{hr}$.

To detect internalized hCD33M, we used anti-FLAG antibody (1:100, Sigma). Briefly cells were permeabilized after fixation step by incubation with $100 \mu \mathrm{L}$ of $5 \%$ goat serum containing $0.1 \%$ Triton $\mathrm{X}-100$ for $5 \mathrm{~min}$ and were further blocked in $5 \%$ goat serum for an additional $5 \mathrm{~min}$. The cells were then incubated with anti-FLAG primary antibody for $1.5 \mathrm{hr}$, followed by centrifugation, and were washed twice with PBS. Cells were incubated with $100 \mu \mathrm{L}$ of AF488conjugated anti mouse IgG1 (1:200) for $1 \mathrm{hr}$, followed by centrifugation and 2 more washed in PBS. Lastly, the cells were incubated with $100 \mu \mathrm{L}$ of Hoechst $(1: 5000$ dilution from a $10 \mathrm{mg} / \mathrm{mL}$ stock in PBS) for 15 min and transferred to glass slides. The extra liquid was gently removed, and cells were cover-slipped with antifade permanent mounting medium (TrueVIEW).

Confocal microscopy was performed with the LSM 700 laser scanning confocal microscope (ZEISS). The images were captured at $63 \mathrm{X}$ magnification and were analyzed with Zen2.6 Black edition software (ZEISS). A total of 12 cells from each condition were imaged for quantification analysis. For quantification, the sum intensity of CD33 signal in each image was normalized to the Hoechst signal.

In vivo administration of CD33L liposomes via intracerebroventricular (ICV) injection. WT and CD33 transgenic animals were anesthetized with isoflurane inhalation, injected with $2 \mathrm{mg} / \mathrm{kg}$ Metacam (analgesic) and immobilized on a stereotaxic device. Stereotaxic marking of the lateral ventricles was performed. Briefly, ICV injections of $1 \mu \mathrm{l}$ CD33L liposome $(20 \mathrm{mM})$ or naked liposome $(20 \mathrm{mM})$ were performed via syringe needle following craniotomy with pre-optimized coordinates (-1.000 medio-lateral; x, -0.300 anterior-posterior, y; and -2.600 dorso-ventral relative to bregma, z) to ensure successful injection inside the right ventricle. Injection was performed over $10 \mathrm{~min}$. After injection animals were kept in a recovery incubator for $30 \mathrm{~min}$, and then they 
were euthanized $4 \mathrm{hr}$ post-injection via CO2. Furthermore, their brain samples were collected and primary microglia were isolated according to the methodology described above. After isolation of microglia from both WT and CD33 transgenic mice, a competitive phagocytosis assay was performed with $200 \mathrm{nM}$ fluorescently labeled aggregated $A \beta$ for 30 min at $37^{\circ} \mathrm{C}$. After phagocytosis cells were stained with an antibody cocktail and the extent of phagocytosis was measured by flow cytometric methods as mentioned above.

\section{Acknowledgment}

M.S.M. thanks funding through GlycoNet, NSERC, CIHR, and a tier II Canada Research Chair in Chemical Glycoimmunology. A.Bh. thanks GlycoNet for a ATOP fellowship. A.Ba. thanks Alberta Innovates for an undergraduate student research fellowship. AESW was supported by endMS MSSoC and WCHRI graduate scholarships and AV by tier II Canada Research Chair in Neural Stem Cell Biology.

\section{References}

(1) Efthymiou, A. G.; Goate, A. M. Late onset Alzheimer's disease genetics implicates microglial pathways in disease risk. Mol Neurodegener 2017, 12 (1), 43.

(2) Sarlus, H.; Heneka, M. T. Microglia in Alzheimer's disease. J Clin Invest 2017, 127 (9), 3240 .

(3) Estus, S.; Shaw, B. C.; Devanney, N.; Katsumata, Y.; Press, E. E.; Fardo, D. W. Evaluation of CD33 as a genetic risk factor for Alzheimer's disease. Acta Neuropathol 2019, 138 (2), 187.

(4) Naj, A. C.; Jun, G.; Beecham, G. W.; Wang, L. S.; Vardarajan, B. N.; Buros, J.; Gallins, P. J.; Buxbaum, J. D.; Jarvik, G. P.; Crane, P. K.et al. Common variants at MS4A4/MS4A6E, CD2AP, CD33 and EPHA1 are associated with late-onset Alzheimer's disease. Nat Genet 2011, 43 (5), 436.

(5) Hollingworth, P.; Harold, D.; Sims, R.; Gerrish, A.; Lambert, J. C.; Carrasquillo, M. M.; Abraham, R.; Hamshere, M. L.; Pahwa, J. S.; Moskvina, V.et al. Common variants at ABCA7, MS4A6A/MS4A4E, EPHA1, CD33 and CD2AP are associated with Alzheimer's disease. Nat Genet 2011, 43 (5), 429.

(6) Malik, M.; Simpson, J. F.; Parikh, I.; Wilfred, B. R.; Fardo, D. W.; Nelson, P. T.; Estus, S. CD33 Alzheimer's risk-altering polymorphism, CD33 expression, and exon 2 splicing. J Neurosci 2013, 33 (33), 13320.

(7) Bhattacherjee, A.; Rodrigues, E.; Jung, J.; Luzentales-Simpson, M.; Enterina, J. R.; Galleguillos, D.; St Laurent, C. D.; Nakhaei-Nejad, M.; Fuchsberger, F. F.; Streith, L.et al. 
Repression of phagocytosis by human CD33 is not conserved with mouse CD33. Commun Biol 2019, 2, 450.

(8) Bhattacherjee, A.; Jung, J.; Zia, S.; Ho, M.; Eskandari-Sedighi, G.; St Laurent, C. D.; McCord, K. A.; Bains, A.; Sidhu, G.; Sarkar, S.et al. The CD33 short isoform is a gain-of-function variant that enhances Abeta1-42 phagocytosis in microglia. Mol Neurodegener 2021, 16 (1), 19.

(9) Ann Butler, C.; Thornton, P.; Charles Brown, G. CD33M inhibits microglial phagocytosis, migration and proliferation, but the Alzheimer's disease-protective variant CD33m stimulates phagocytosis and proliferation, and inhibits adhesion. J Neurochem 2021, DOI:10.1111/jnc.15349 10.1111/jnc.15349.

(10) Wissfeld, J.; Nozaki, I.; Mathews, M.; Raschka, T.; Ebeling, C.; Hornung, V.; Brustle, O.; Neumann, H. Deletion of Alzheimer's disease-associated CD33 results in an inflammatory human microglia phenotype. Glia 2021, 69 (6), 1393.

(11) Price, B. R. Preclinical Targeting of TREM2 for the Treatment of Alzheimer's Disease-type Pathology in a Transgenic Mouse Model. 2019.

(12) Schlepckow, K.; Monroe, K. M.; Kleinberger, G.; Cantuti-Castelvetri, L.; Parhizkar, S.; Xia, D.; Willem, M.; Werner, G.; Pettkus, N.; Brunner, B.et al. Enhancing protective microglial activities with a dual function TREM2 antibody to the stalk region. EMBO Mol Med 2020, 12 (4), e11227.

(13) Fassler, M.; Rappaport, M. S.; Cuno, C. B.; George, J. Engagement of TREM2 by a novel monoclonal antibody induces activation of microglia and improves cognitive function in Alzheimer's disease models. J Neuroinflammation 2021, 18 (1), 19.

(14) Griciuc, A.; Patel, S.; Federico, A. N.; Choi, S. H.; Innes, B. J.; Oram, M. K.; Cereghetti, G.; McGinty, D.; Anselmo, A.; Sadreyev, R. I.et al. TREM2 Acts Downstream of CD33 in Modulating Microglial Pathology in Alzheimer's Disease. Neuron 2019, 103 (5), 820.

(15) Bradshaw, E. M.; Chibnik, L. B.; Keenan, B. T.; Ottoboni, L.; Raj, T.; Tang, A.; Rosenkrantz, L. L.; Imboywa, S.; Lee, M.; Von Korff, A.et al. CD33 Alzheimer's disease locus: altered monocyte function and amyloid biology. Nat Neurosci 2013, 16 (7), 848.

(16) Walter, R. B. Expanding use of CD33-directed immunotherapy. Expert Opin Biol Ther 2020, 20 (9), 955.

(17) Malik, M.; Chiles, J., 3rd; Xi, H. S.; Medway, C.; Simpson, J.; Potluri, S.; Howard, D.; Liang, Y.; Paumi, C. M.; Mukherjee, S.et al. Genetics of CD33 in Alzheimer's disease and acute myeloid leukemia. Hum Mol Genet 2015, 24 (12), 3557.

(18) Walter, R. B.; Appelbaum, F. R.; Estey, E. H.; Bernstein, I. D. Acute myeloid leukemia stem cells and CD33-targeted immunotherapy. Blood 2012, 119 (26), 6198.

(19) Walter, R. B.; Raden, B. W.; Kamikura, D. M.; Cooper, J. A.; Bernstein, I. D. Influence of CD33 expression levels and ITIM-dependent internalization on gemtuzumab ozogamicin-induced cytotoxicity. Blood 2005, 105 (3), 1295.

(20) Walter, R. B.; Raden, B. W.; Zeng, R.; Hausermann, P.; Bernstein, I. D.; Cooper, J. A. ITIM-dependent endocytosis of CD33-related Siglecs: role of intracellular domain, tyrosine phosphorylation, and the tyrosine phosphatases, Shp1 and Shp2. J Leukoc Biol 2008, 83 (1), 200.

(21) Macauley, M. S.; Crocker, P. R.; Paulson, J. C. Siglec-mediated regulation of immune cell function in disease. Nat Rev Immunol 2014, 14 (10), 653.

(22) Rodrigues, E.; Jung, J.; Park, H.; Loo, C.; Soukhtehzari, S.; Kitova, E. N.; Mozaneh, F.; Daskhan, G.; Schmidt, E. N.; Aghanya, V.et al. A versatile soluble siglec scaffold for sensitive and quantitative detection of glycan ligands. Nat Commun 2020, 11 (1), 5091.

(23) Bull, C.; Nason, R.; Sun, L.; Van Coillie, J.; Madriz Sorensen, D.; Moons, S. J.; Yang, Z.; Arbitman, S.; Fernandes, S. M.; Furukawa, S.et al. Probing the binding specificities of human Siglecs by cell-based glycan arrays. Proc Natl Acad Sci U S A 2021, 118 (17).

(24) Angata, T.; Nycholat, C. M.; Macauley, M. S. Therapeutic Targeting of Siglecs using Antibody- and Glycan-Based Approaches. Trends Pharmacol Sci 2015, 36 (10), 645. 
(25) Duan, S.; Paulson, J. C. Siglecs as Immune Cell Checkpoints in Disease. Annu Rev Immunol 2020, 38, 365.

(26) Lenza, M. P.; Atxabal, U.; Oyenarte, I.; Jimenez-Barbero, J.; Ereno-Orbea, J. Current Status on Therapeutic Molecules Targeting Siglec Receptors. Cells 2020, 9 (12).

(27) Movsisyan, L. D.; Macauley, M. S. Structural advances of Siglecs: insight into synthetic glycan ligands for immunomodulation. Org Biomol Chem 2020, 18 (30), 5784.

(28) Smith, B. A. H.; Bertozzi, C. R. The clinical impact of glycobiology: targeting selectins, Siglecs and mammalian glycans. Nat Rev Drug Discov 2021, 20 (3), 217.

(29) Rillahan, C. D.; Macauley, M. S.; Schwartz, E.; He, Y.; McBride, R.; Arlian, B. M.; Rangarajan, J.; Fokin, V. V.; Paulson, J. C. Disubstituted Sialic Acid Ligands Targeting Siglecs CD33 and CD22 Associated with Myeloid Leukaemias and B Cell Lymphomas. Chem Sci 2014, $5(6), 2398$.

(30) Miles, L. A.; Hermans, S. J.; Crespi, G. A. N.; Gooi, J. H.; Doughty, L.; Nero, T. L.; Markulic, J.; Ebneth, A.; Wroblowski, B.; Oehlrich, D.et al. Small Molecule Binding to Alzheimer Risk Factor CD33 Promotes Abeta Phagocytosis. iScience 2019, 19, 110.

(31) Knop, K.; Hoogenboom, R.; Fischer, D.; Schubert, U. S. Poly(ethylene glycol) in drug delivery: pros and cons as well as potential alternatives. Angew Chem Int Ed Engl 2010, 49 (36), 6288.

(32) Duan, S.; Koziol-White, C. J.; Jester, W. F., Jr.; Smith, S. A.; Nycholat, C. M.; Macauley, M. S.; Panettieri, R. A., Jr.; Paulson, J. C. CD33 recruitment inhibits IgE-mediated anaphylaxis and desensitizes mast cells to allergen. J Clin Invest 2019, 129 (3), 1387.

(33) Jusu, S. M.; Obayemi, J. D.; Salifu, A. A.; Nwazojie, C. C.; Uzonwanne, V.; Odusanya, O. S.; Soboyejo, W. O. Drug-encapsulated blend of PLGA-PEG microspheres: in vitro and in vivo study of the effects of localized/targeted drug delivery on the treatment of triple-negative breast cancer. Sci Rep 2020, 10 (1), 14188.

(34) Pluvinage, J. V.; Haney, M. S.; Smith, B. A. H.; Sun, J.; Iram, T.; Bonanno, L.; Li, L.; Lee, D. P.; Morgens, D. W.; Yang, A. C.et al. CD22 blockade restores homeostatic microglial phagocytosis in ageing brains. Nature 2019, 568 (7751), 187.

(35) Biedermann, B.; Gil, D.; Bowen, D. T.; Crocker, P. R. Analysis of the CD33-related siglec family reveals that Siglec-9 is an endocytic receptor expressed on subsets of acute myeloid leukemia cells and absent from normal hematopoietic progenitors. Leuk Res 2007, 31 (2), 211.

(36) Perez-Oliva, A. B.; Martinez-Esparza, M.; Vicente-Fernandez, J. J.; Corral-San Miguel, R.; Garcia-Penarrubia, P.; Hernandez-Caselles, T. Epitope mapping, expression and posttranslational modifications of two isoforms of CD33 (CD33M and CD33m) on lymphoid and myeloid human cells. Glycobiology 2011, 21 (6), 757.

(37) Paul, S. P.; Taylor, L. S.; Stansbury, E. K.; McVicar, D. W. Myeloid specific human CD33 is an inhibitory receptor with differential ITIM function in recruiting the phosphatases SHP-1 and SHP-2. Blood 2000, 96 (2), 483.

(38) Paoli, E. E.; Ingham, E. S.; Zhang, H.; Mahakian, L. M.; Fite, B. Z.; Gagnon, M. K.; Tam, S.; Kheirolomoom, A.; Cardiff, R. D.; Ferrara, K. W. Accumulation, internalization and therapeutic efficacy of neuropilin-1-targeted liposomes. J Control Release 2014, 178, 108.

(39) Wang, X.; Quu, Y.; Wang, M.; Zhang, C.; Zhang, T.; Zhou, H.; Zhao, W.; Zhao, W.; Xia, G.; Shao, R. Endocytosis and Organelle Targeting of Nanomedicines in Cancer Therapy. Int $J$ Nanomedicine 2020, 15, 9447.

(40) Kim, B.; Shin, J.; Kiziltepe, T.; Bilgicer, B. Identification of a moderate affinity CD22 binding peptide and in vitro optimization of peptide-targeted nanoparticles for selective uptake by CD22+ B-cell malignancies. Nanoscale 2020, 12 (21), 11672.

(41) Macauley, M. S.; Pfrengle, F.; Rademacher, C.; Nycholat, C. M.; Gale, A. J.; von Drygalski, A.; Paulson, J. C. Antigenic liposomes displaying CD22 ligands induce antigen-specific B cell apoptosis. J Clin Invest 2013, 123 (7), 3074. 
(42) Delaveris, C. S.; Chiu, S. H.; Riley, N. M.; Bertozzi, C. R. Modulation of immune cell reactivity with cis-binding Siglec agonists. Proc Natl Acad Sci U S A 2021, 118 (3).

(43) Vieira, D. B.; Gamarra, L. F. Getting into the brain: liposome-based strategies for effective drug delivery across the blood-brain barrier. Int $J$ Nanomedicine 2016, 11, 5381.

(44) Kohane, D. S. Microparticles and nanoparticles for drug delivery. Biotechnol Bioeng 2007, 96 (2), 203.

(45) De Jong, W. H.; Borm, P. J. Drug delivery and nanoparticles:applications and hazards. Int J Nanomedicine 2008, 3 (2), 133.

(46) Ostrowitzki, S.; Lasser, R. A.; Dorflinger, E.; Scheltens, P.; Barkhof, F.; Nikolcheva, T.; Ashford, E.; Retout, S.; Hofmann, C.; Delmar, P.et al. A phase III randomized trial of gantenerumab in prodromal Alzheimer's disease. Alzheimers Res Ther 2017, 9 (1), 95.

(47) van Dyck, C. H. Anti-Amyloid-beta Monoclonal Antibodies for Alzheimer's Disease: Pitfalls and Promise. Biol Psychiatry 2018, 83 (4), 311.

(48) The Lancet, N. Solanezumab: too late in mild Alzheimer's disease? Lancet Neurol 2017, $16(2), 97$.

(49) Salloway, S.; Honigberg, L. A.; Cho, W.; Ward, M.; Friesenhahn, M.; Brunstein, F.; Quartino, A.; Clayton, D.; Mortensen, D.; Bittner, T.et al. Amyloid positron emission tomography and cerebrospinal fluid results from a crenezumab anti-amyloid-beta antibody double-blind, placebo-controlled, randomized phase II study in mild-to-moderate Alzheimer's disease (BLAZE). Alzheimers Res Ther 2018, 10 (1), 96.

(50) Spencer, B.; Masliah, E. Immunotherapy for Alzheimer's disease: past, present and future. Front Aging Neurosci 2014, 6, 114.

(51) Pardridge, W. M. Blood-Brain Barrier and Delivery of Protein and Gene Therapeutics to Brain. Front Aging Neurosci 2019, 11, 373.

(52) Kim, M. Y.; Yu, K. R.; Kenderian, S. S.; Ruella, M.; Chen, S.; Shin, T. H.; Aljanahi, A. A.; Schreeder, D.; Klichinsky, M.; Shestova, O.et al. Genetic Inactivation of CD33 in Hematopoietic Stem Cells to Enable CAR T Cell Immunotherapy for Acute Myeloid Leukemia. Cell 2018, 173 (6), 1439. 